\title{
Review
}

Hyeongwoo Lee, Dong Yun Lee, Min Gu Kang, Yeonjeong Koo, Taehyun Kim and Kyoung-Duck Park*

\section{Tip-enhanced photoluminescence nano-spectroscopy and nano-imaging}

https://doi.org/10.1515/nanoph-2020-0079

Received February 2, 2020; accepted April 9, 2020; published online June 3, 2020

\begin{abstract}
Photoluminescence (PL), a photo-excited spontaneous emission process, provides a wealth of optical and electronic properties of materials, which enable microscopic and spectroscopic imaging, biomedical sensing and diagnosis, and a range of photonic device applications. However, conventional far-field PL measurements have limitations in sensitivity and spatial resolution, especially to investigate single nano-materials or nano-scale dimension of them. In contrast, tip-enhanced photoluminescence (TEPL) nano-spectroscopy provides an extremely high sensitivity with $<10 \mathrm{~nm}$ spatial resolution, which allows the desired nano-scale characterizations. With outstanding and unique optical properties, low-dimensional quantum materials have recently attracted much attention, and TEPL characterizations, i. e., probing and imaging, and even control at the nanoscale, have been extensively studied. In this review, we discuss the fundamental working mechanism of PL enhancement by plasmonic tip, and then highlight recent advances in TEPL studies for low-dimensional quantum materials. Finally, we discuss several remaining challenges of TEPL nano-spectroscopy and nano-imaging, such as implementation in non-ambient media and in situ environments, limitations in sample structure, and control of near-field polarization, with perspectives of the approach and its applications.
\end{abstract}

Keywords: low-dimensional quantum materials; plasmonic tip; tip-enhanced photoluminescence (TEPL).

Hyeongwoo Lee and Dong Yun Lee are equally contributed.

*Corresponding author: Kyoung-Duck Park, Department of Physics, Ulsan National Institute of Science and Technology (UNIST), Ulsan, 44919, South Korea, E-mail: kdpark@unist.ac.kr. https://orcid.org/ 0000-0002-9302-9384

Hyeongwoo Lee, Dong Yun Lee, Min Gu Kang, Yeonjeong Koo and Taehyun Kim: Department of Physics, Ulsan National Institute of Science and Technology (UNIST), Ulsan, 44919, South Korea

\section{Introduction}

With fundamental understanding as well as engineering studies for a range of organic and inorganic materials and hard and soft matter systems, we have achieved remarkable developments in modern device technologies. While we mainly used bulk material systems for device applications in the past several decades [1], they have reached the limit in their electrical and optical properties, device performance, and application range. To overcome these limitations in bulk systems, on the one hand, a significant effort is being made to discover unknown materials which show superior performance [2,3]. On the other hand, dimensional reduction of conventional bulk systems gives rise to new physical phenomena, such as quantum confinement effect [4] and dielectric screening effect [5], which lead to significantly improved functions and properties $[6,7]$. Therefore, low-dimensional quantum materials have emerged as appealing resources for the next generation devices [8].

As the physical length scale of quantum materials is reduced to the nano-scale, understanding physical properties at their natural scale is desirable. To investigate structural properties of low-dimensional quantum materials, e. g., surface roughness, lattice structure, and structural defects, scanning tunneling microscopy (STM) and transmission electron microscopy (TEM) are widely used [9-13]. Specifically, STM is a unique local probe providing electrical properties $[10,11,14]$ and spin information [15-17] with atomic resolution. However, measurements are difficult in ambient condition and careful sample preparation is needed to obtain the desired information with high resolution [18]. In addition, optical properties associated with electronic processes, such as absorption and photoluminescence (PL), cannot be investigated with STM and TEM.

In contrast, confocal microscopy combined with PL spectroscopy allows to investigate these optical responses in ambient condition, yet with diffraction limited spatial resolution and low sensitivity [19]. To this end, near-field PL measurement approach, i. e., tip-enhanced 
PL (TEPL) nano-spectroscopy and -imaging [20], has emerged for multifunctional nano-characterizations of low-dimensional quantum materials, since it provides high spatial resolution of $\sim 10 \mathrm{~nm}$ with high optical sensitivity owing to the plasmonic nano-optical antenna effect $[21,22]$.

In this review, we briefly describe technical limitations in far-field optical imaging and summarize near-field imaging methods from aperture fiber type approach to tipenhanced nano-spectroscopy and -imaging. Further, we describe the principles and enhancement factor of TEPL. We then discuss nano-scale field localization at the apex of plasmonic tip in various conditions, e. g., materials of a tip, substrates, and polarization, based on electromagnetic simulation results. Next, we focus on highlighting recent TEPL studies for low-dimensional materials, specifically applications to 0-dimensional (OD), 1D, and 2D quantum systems. Lastly, we discuss several remaining challenges of TEPL nano-spectroscopy and nano-imaging, such as implementation in non-ambient media and in situ environments, limitations in sample structure, and control of near-field polarization, with perspectives of the approach and its further applications.

\section{Historical developments of near- field microscopies}

In far-field spectroscopy and imaging, the Airy patterns from two different objects are distinct and resolvable if they are apart farther than the Rayleigh criterion [23]. To overcome this diffraction limit $(\lambda / 2 \mathrm{NA}$, where $\lambda$ is a wavelength of light and NA is a numerical aperture of lens), near-field optical imaging techniques have emerged. The original theoretical concept of high resolution near-field imaging was proposed by E. H. Synge in 1928 [24]. He proposed to make a small aperture (diameter of $<10 \mathrm{~nm}$ ) in thin metal film and use the transmitted light through that aperture to illuminate a sample placed near the nano-aperture. However, this innovative idea could not be practically implemented for a long time due to its several technological difficulties at that time, such as nano-fabrication, nano-precision positioning, and low sensitivity of an optical detector. Almost 60 years later, this original idea of near-field imaging had been implemented by the advent of a scanning probe microscopy (SPM) technique [25, 26]. Near-field scanning optical microscopy (NSOM) is the first approach for measuring near-field optical responses using an optical fiber probe.
The metal-coated optical fiber probe has a nano-aperture with a diameter of $\sim 50-100 \mathrm{~nm}$, which determines a spatial resolution of NSOM, to detect or to illuminate near-field signals on the surface of samples [27]. With a high spatial resolution far beyond the diffraction limit, many groundbreaking works were allowed in physics [28, 29], chemistry [30, 31], biology [32, 33], and material sciences [34-37].

However, against all expectations, the application range of NSOM had been highly limited due to the low sensitivity, since the transmission intensity through the NSOM probe is inversely proportional to the fourth power of the aperture size [38, 39]. In addition, other problems of optical fibers, such as wavelength-selective optical loss, polarization modification, and dispersion, also restricted the broad applications of NSOM. To overcome these constraints of an optical fiber probe, an inverse approach using near-field scattering, so called apertureless NSOM (ANSOM) or scattering-scanning near-field optical microscopy (s-SNOM), was proposed [29, 40-44]. In this method, evanescent waves on the surface of a sample are scattered by an apertureless tip and converted into propagating waves, which provide a near-field image with a spatial resolution of $<10 \mathrm{~nm}$ in optimized conditions. This has been a great step forward, but weak near-field scattering signal was still a significant issue because image contrast and spatial resolution can be easily deteriorated due to the farfield background.

At that time, plasmonic nano-materials $(\mathrm{Au}$ or $\mathrm{Ag}$ in the visible frequency) had gained great attention with surface enhanced Raman scattering (SERS) studies, which showed single-molecule sensitivity [45, 46]. Similarly, plasmonic tip has started to be used in $s$-SNOM to obtain the desired strong near-field signals. The first demonstration was tip-enhanced Raman spectroscopy (TERS) [47-51] and this tip-enhanced nano-spectroscopy and -imaging approach was extended widely to detect elastic [52, 53] and other inelastic scattering $[54,55]$, as well as nonlinear optical responses [56, 57]. Specifically, TEPL spectroscopy provides electronic properties of semiconducting materials at the nano-scale, such as quantum dots [58], quantum wires [59], and transition metal dichalcogenides (TMDs) [20]. Most studies showed highly enhanced PL signals, yet in the weak coupling regime. Recently, TEPL approach further advanced to tip-enhanced strong coupling (TESC) spectroscopy using plasmonic tip-cavity at room temperature [60]. In brief, historical development in near-field microscopy is summarized in Figure 1 and we focus on TEPL spectroscopy and its applications in this review. 


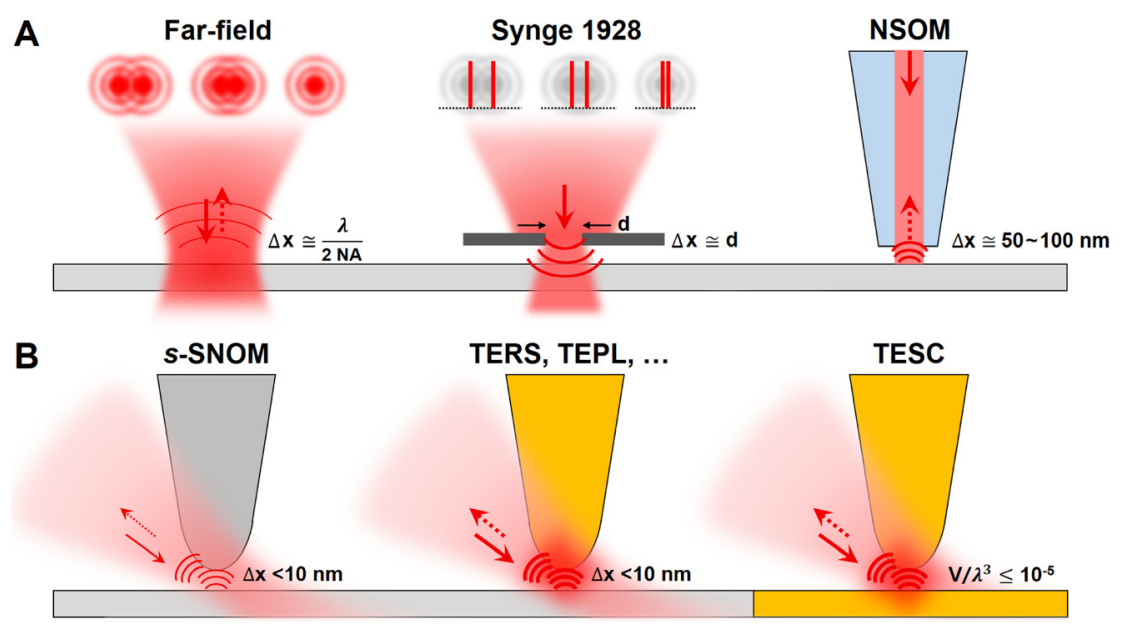

Figure 1: $(A)$ The brief experimental schematics with spatial resolution for farfield optical microscope, Synge's theoretical proposal, and near-field scanning optical microscopy (NSOM). $\Delta x$ denotes spatial resolution. (B) Schematics of scattering-type scanning near-field optical microscopy (s-SNOM), tip-enhanced nano-spectroscopy (TERS and TEPL), and tip-enhanced strong coupling (TESC) spectroscopy [37]. $V / \lambda^{3}$ denotes mode volume for an excitation wavelength.

\section{TEPL mechanism and enhancement factor}

Basically, field enhancement in plasmonic tips is attributed to two phenomena as follows. First, electric charges are highly concentrated in the vicinity of a plasmonic tip due to the electrostatic lightning rod effect [61]. Second, when an external electromagnetic field, e. g., excitation laser beam, is applied to the tip, optical fields are strongly localized at the apex due to the collective resonant oscillations of the electrons, i. e., localized surface plasmon resonance (LSPR) effect [62].

The LSPR of plasmonic tip can be understood with simple analytical models $[37,63,58]$. The tip is approximated as a polarizable sphere with a radius $r(\ll \lambda)$ and frequency-dependent permittivity $\epsilon_{s p h}[\omega]$. Based on the Clausius-Mossotti relation, the induced polarization of the sphere is defined by $\mathbf{p}_{s p h}=\alpha_{s p h} \epsilon_{0} \mathbf{E}_{i n c}$, where $\mathbf{E}_{i n c}$ is the incident electric field. The polarizability $\alpha_{s p h}$ is given by

$$
\alpha_{s p h}=4 \pi r^{3}\left(\frac{\epsilon_{s p h}[\omega]-1}{\epsilon_{s p h}[\omega]+2}\right) .
$$

Since the permittivity of $\mathrm{Ag}$ and $\mathrm{Au}$ nano-spheres makes the denominator of Eq. (1) to be very small in the visible frequency, the polarizability, scattering cross-section $\left(\sigma_{\text {scat }} \propto \alpha_{\text {sph }}^{2}\right)$, and the induced polarization show resonance behaviors with nano-optical antenna effect.

When a plasmonic tip with $\mathbf{E}_{i n c, z}$, approaches closely to a sample or a substrate with a distance $d$, the polarizability is understood with the coupled dipole model $[37,63,64]$. If we assume the sphere as a point dipole with the dipole orientation in the $z$-axis (perpendicular to the sample surface), an image dipole associated with the localized surface charge is induced beneath the surface at $-d$ with the dielectric function of $\beta_{\text {surf }}=\left(\epsilon_{s p h}[\omega]-1\right) /$ $\left(\epsilon_{s p h}[\omega]+1\right)$. Hence, the effective polarizability $\alpha_{e f f}$ and polarization $\mathbf{p}_{\text {eff }}$ due to the dipole-dipole interaction can be expressed as

$$
\begin{gathered}
\alpha_{e f f}=\frac{\alpha_{s p h}\left(1+\beta_{\text {surf }}\right)}{1-\alpha_{\text {sph }} \beta_{\text {surf }} /\left(16 \pi(r+d)^{3}\right)}, \\
\mathbf{p}_{\text {eff }}=\alpha_{e f f} \epsilon_{0} \mathbf{E}_{\text {inc }, z} .
\end{gathered}
$$

The near-field localization at the apex of plasmonic tip leads to much larger excitation rate for PL response of the sample compared to that of conventional PL spectroscopy. In addition to the excitation rate, the spontaneous emission rate of the sample is also increased by the presence of plasmonic tip close to an emitter, known as the Purcell effect $F_{p}=\left(3 / 4 \pi^{2}\right)(\lambda / n)^{3} Q / V$, where $n$ is index of refraction of an optical cavity with the quality factor $Q$ and mode volume $V$ [65]. Even though a non-radiative energy transfer from the emitter to the plasmonic tip [66], i. e., PL quenching, occasionally occurs due to ohmic losses in the metal, PL signal is generally enhanced by the plasmonic tip effect [67].

The enhancement factor $E F$ of TEPL is estimated with contributions of the excitation rate and the spontaneous emission rate [68]. In general, PL intensity is quantified by the product of the excitation rate $\Gamma^{e x}$ and the quantum efficiency $\eta$. The $\eta$ can be expressed as $\eta=k^{r a d} /\left(k^{r a d}+k^{n r a d}\right)$, where $k^{r a d}$ is the radiative decay rate and $k^{\text {nrad }}$ is the nonradiative decay rate. Accordingly, $E F$ can be defined as the ratio between TEPL intensity and far-field PL intensity, given by 


$$
E F=\frac{\Gamma_{T E}^{e x}}{\Gamma_{F F}^{e x}} \cdot \frac{\eta_{T E}}{\eta_{F F}}
$$

where subscripts $T E$ and $F F$ denote tip-enhancement and far-field. Tip-enhanced excitation rate can also be expressed as $\Gamma_{T E}^{e x} \cong\left(E_{T E} / E_{F F}\right)^{2}$ with tip-enhanced electric field intensity. Note that TEPL enhancement was reported as high as $\sim 6 \times 10^{5}$ with Au tip-cavity for dark excitons in $\mathrm{WSe}_{2}$ monolayer [69].

\section{Field enhancement in plasmonic tips}

Field enhancement in plasmonic tips leads to the large excitation rate for strong TERS and TEPL responses, which provide high spatial resolution imaging. To quantify the enhanced field distribution at the tip apex, there have been numerous theoretical studies [70-75]. In this section, we discuss field distribution and enhancement in various conditions, e. g., tip material, substrate, and excitation polarization, with electromagnetic simulation results performed by finite difference time domain (FDTD) or finite element method (FEM).

Photo-induced electronic responses of plasmonic tip are described by the dielectric function [76]. When an external field is applied to a plasmonic tip, the real part of the dielectric function describes the polarization strength as a function of the excitation frequency, i. e., it determines a spectral resonance of the LSPR. The imaginary part describes the optical losses, which determine the plasmon bandwidth [77-79]. Silver and gold tips are commonly used for TERS and TEPL spectroscopy due to their low losses in the visible and near infrared frequencies [70, 80-82]. Under the side-illumination with $p$-polarized light (E-field parallel with respect to the tipaxis), optical field is strongly localized at the apex of plasmonic tip owing to the lightning rod effect [71, 83-88]. Since free electrons of a metal tip resonantly oscillate with excitation field perpendicular to the surface, optical nearfields of $\mathrm{E}_{z}$ and $\mathrm{E}_{x}$ components are distributed at the tip apex, as shown in Figure 2A and B. Figure $2 \mathrm{C}$ shows the simulated optical field distribution at the apex of tips made with different materials, such as $\mathrm{Au}, \mathrm{W}, \mathrm{Si}$, and glass [70]. Au tip shows the highest field enhancement due to the LSPR effect. On the other hand, tungsten (W) tip shows low field enhancement, which is comparable to $\mathrm{Si}$ and glass tips, due to the strong damping of the field caused by absorptive loss in a poor conductor.

The geometry of a plasmonic tip, such as the apex size and the cone angle, influences the field enhancement and plasmon resonance. Figure 2D shows the calculated field enhancement of Au tip at a $630 \mathrm{~nm}$ excitation as a function of the tip-radius and the cone angle [70]. The field enhancement is gradually increased ranging from 10 to $10^{3}$ times as the radius is decreased for different cone angles. Decreasing cone angle also gives rise to stronger field enhancement. In contrast, an opposite simulation result was reported by Zhang et al. [72]. They modeled the geometry of Ag tip with infinite length and the fixed tip-radius of $20 \mathrm{~nm}$, and simulated the field enhancement of the tip with cone angles of $15^{\circ}, 20^{\circ}$, and $25^{\circ}$. As can be seen in Figure 2E, tips with larger cone angle show higher field enhancement in the visible and near infrared regions. This behavior is similar to the field enhancement of biconical antennas [89]. A more sophisticated simulation with respect to both the cone angle and the excitation wavelength was performed by Goncharenko et al. [73]. Figure 2F shows the spectral and cone angle dependence of the field enhancement for Ag tip. For the increasing cone angle, the plsamon resonance is blue-shifted with the increasing field enhancement. However, at the normal excitation wavelength of $550 \sim 650 \mathrm{~nm}$ for TERS and TEPL, sharp tips give
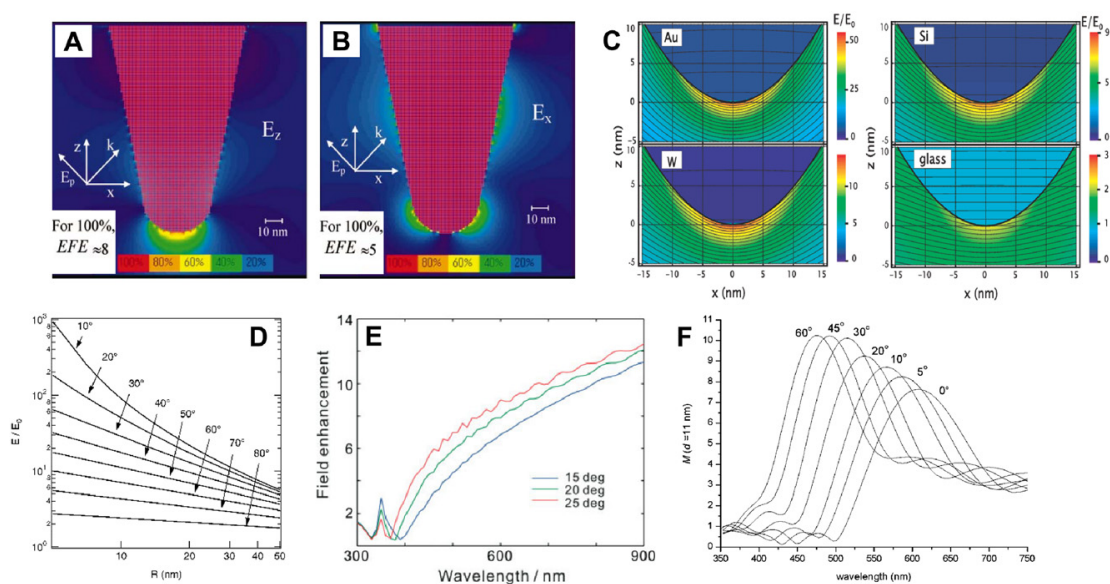

Figure 2: (A-B) Simulated $E_{z}$ and $E_{x}$ field distributions of Au tip illuminated by $p$ polarized light [71]. Copyright 2004 Optical Society of America. (C) Simulated electric field distribution of free-standing tips made with various materials, such as $\mathrm{Au}, \mathrm{W}, \mathrm{Si}$, and glass [70]. (D-F) Simulated field enhancement of plasmonic tips with respect to the tip-radius, cone angle, and excitation wavelength $[70,72,73]$. Reprinted with permission from [70]. Copyright (2008) American Chemical Society. Copyright 2009 John Wiley \& Sons, Ltd [72]. Reprinted from [73], with the permission of AIP Publishing. 
the higher field enhancement. These inconsistent simulation results $[70,72,73]$ of cone angle dependence are mainly due to the complex interplay of competing mechanisms on the surface charge density. When the cone angle is increased, on the one hand, the surface charge density and field enhancement are increased due to the decreased value of the imaginary part of the metal dielectric function. On the other hand, larger cone angle leads to enlarging the surface area, which reduces the charge density and field enhancement [73].

As we described in section 3, a substrate plays an important role for the field enhancement due to the dipoledipole interaction [70, 72, 74, 75, 90-92]. Figure 3A shows a variation of the field enhancement for different distances ranging from 2 to $40 \mathrm{~nm}$ between $\mathrm{Au}$ tip and Au substrate [70]. In this simulation, the tip-radius is set to $10 \mathrm{~nm}$ and an excitation wavelength of $630 \mathrm{~nm}$ is used. The Au surface is fixed at $z=0 \mathrm{~nm}$ and the variable tip position is located at $z=d$ positions. The field enhancement is dramatically increased when the tip-substrate distance is decreased to a few nanometers because the effective polarizability is inversely proportional to the third power of the distance $d$, as can be seen in Eq. (2). Specifically, the near-field interaction becomes effective when the tip-substrate distance is shorter than twice the radius of tip. As for the spatial distribution, the field localization is stronger near the tip apex compared to the Au surface based on the tip geometry and the coupled dipole model. Figure 3B clearly demonstrates the lateral spatial distribution of the localized field. The curves show lateral cross-sections of the enhanced optical field near the $\mathrm{Au}$ surface for different tip-substrate distances. This result indicates that the tip-substrate distance significantly influences the spatial resolution in near-field imaging. Table 1 shows the simulated field enhancement in the plasmonic gap between $\mathrm{Ag}$ tip and different substrates [74]. As expected, good conductor substrates give stronger field enhancement due to the higher surface charge density.

In addition to the tip-material, geometry, and substrate effects, the field enhancement is also affected by the propagation direction and polarization of the excitation field [75]. Figure 4 shows optical field intensity distribution at the apex of Ag tip at an excitation wavelength of $532 \mathrm{~nm}$ for different polarization conditions. When the excitation polarization is perpendicular with respect to the tip-axis, the field enhancement on the tip shaft is more significant compared to the tip apex as shown in Figure 4A. In contrast, for the excitation polarization in parallel with the tip-axis, optical near-fields are strongly localized at the apex with $\sim 10$ times larger field intensity (Figure 4C). Figure $4 \mathrm{~B}$ and $\mathrm{D}$ shows stronger field enhancement and localization due to the substrate effect, as we discussed
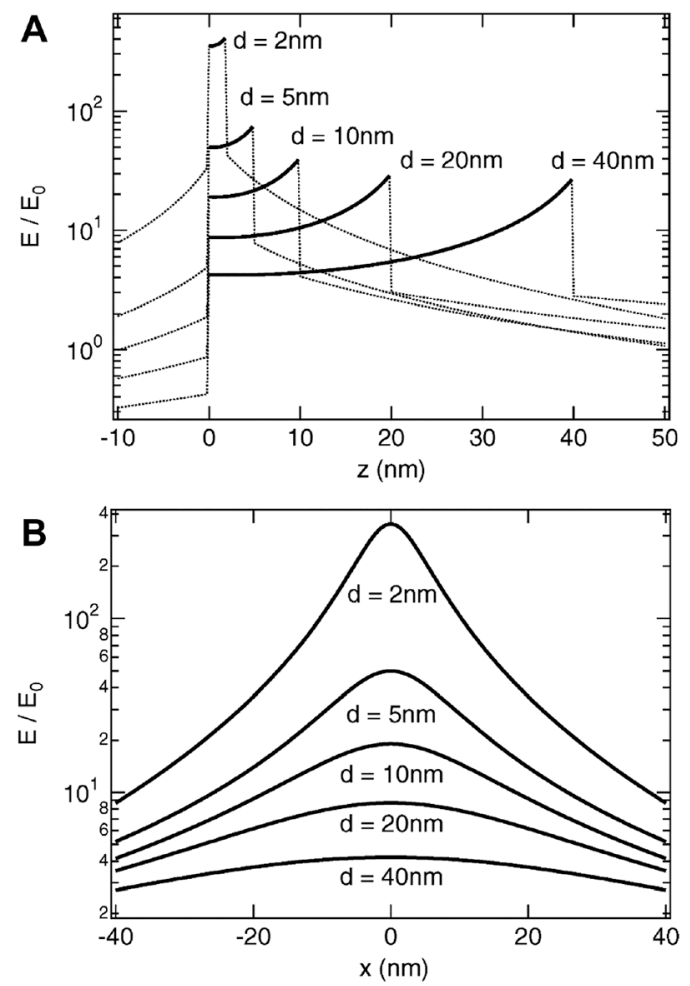

Figure 3: (A) Simulated variation of the field enhancement for different distances ranging from 2 to $40 \mathrm{~nm}$ between Au tip and Au substrate. (B) Simulated lateral cross-sections of the enhanced optical field near the Au surface for different tip-substrate distances. Reprinted with permission from [70]. Copyright (2008) American Chemical Society.

earlier. Based on this feature, the side-illumination geometry is generally used for TERS [93-96] and TEPL [20] experiments. Some research groups use the bottomillumination geometry to measure transparent samples [53, 97]. In this case, since the excitation direction is fixed along to the tip-axis, a linearly polarized excitation beam is not suitable for the large field enhancement, as can be seen in Figure 4A. Therefore, a radially polarized beam is used to effectively excite plasmonic tip. Figure $4 \mathrm{E}$ and $\mathrm{F}$ shows the field intensity distribution with a radially polarized light from the bottom. In this case, the enhancement factor is much higher than the side-illumination case using a linearly polarized light because the excitation field is more effectively spatially localized near the tip, as also can be seen in far-field studies [98-102]. Note that the field enhancement factor can be additionally increased by using the tilted tip geometry [57].

\section{Applications to OD materials}

Weak optical responses from 0D materials, such as single molecules and nano-crystals, are generally hard to detect 
Table 1: Simulated field enhancement in the plasmonic gap between Ag tip and different substrates. FE: finite element, BCB: brilliant cresyl blue [74]. Copyright 2013 by John Wiley \& Sons, Inc. Reprinted by permission of John Wiley \& Sons, Inc.

\begin{tabular}{|c|c|c|c|c|c|}
\hline Metal & $E_{2}$ (FDTD) & $E_{4}$ (FDTD) & $E_{2}(\mathrm{FE})$ & TERS (BCB) & TERS (PhS) \\
\hline $\mathrm{Au}$ & 1 & 1 & 1 & 1 & 1 \\
\hline $\mathrm{Cu}$ & 0.92 & 0.848 & & $0.16 \pm 0.11$ & \\
\hline $\mathrm{Ag}$ & 0.81 & 0.654 & 0.559 & $0.23 \pm 0.05$ & 0.77 \\
\hline Al & 0.63 & 0.402 & & & \\
\hline $\mathrm{Pd}$ & 0.61 & 0.375 & & & \\
\hline $\mathrm{Pt}$ & 0.55 & 0.297 & & & \\
\hline $\mathrm{Ni}$ & 0.53 & 0.281 & & $0.07 \pm 0.06$ & 0.72 \\
\hline $\mathrm{Cr}$ & 0.51 & 0.264 & & & \\
\hline $\mathrm{Ti}$ & 0.45 & 0.199 & & $0.14 \pm 0.12$ & \\
\hline Mo & 0.44 & 0.194 & & $0.16 \pm 0.11$ & \\
\hline W & 0.41 & 0.166 & & & \\
\hline $\mathrm{Al}_{2} \mathrm{O}_{3}$ name & 0.09 & & & & \\
\hline $\mathrm{SiO}_{2}$ nam & 0.05 & 0.003 & & & \\
\hline Tip only & 0.05 & 0.003 & & & \\
\hline
\end{tabular}

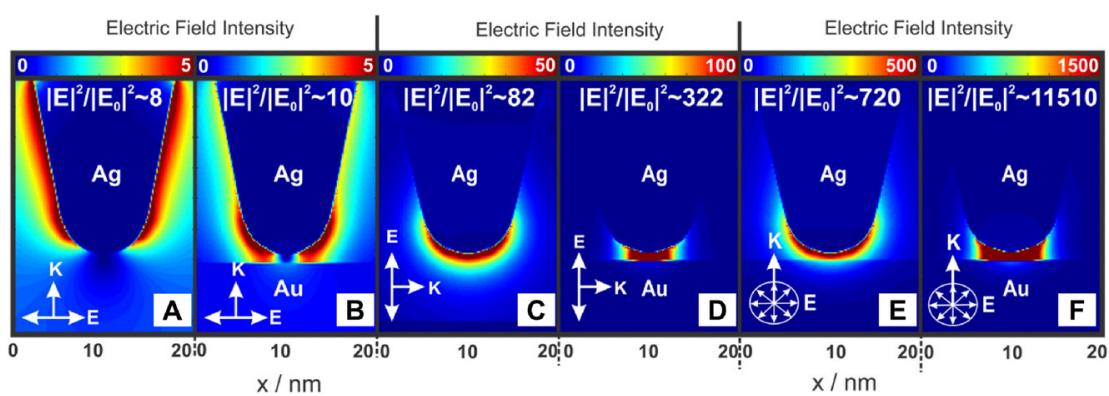

Figure 4: Electric field intensity distribution at the apex of Ag tip at an excitation wavelength of $532 \mathrm{~nm}$ for different polarization conditions. The bottomillumination with a linearly polarized light without (A) and with Au substrate (B). The side-illumination with a linearly polarized light without (C) and with Au substrate (D). The bottom-illumination with a radially polarized light without $(\mathrm{E})$ and with $\mathrm{Au}$ substrate (F) [75]. Copyright (2013) Optical Society of America.

with conventional optical microscopy $[103,104]$. While a quantum dot, $0 \mathrm{D}$ semiconducting material which has smaller diameter than exciton Bohr radius, gives strong PL responses [105], characterizing intrinsic optical properties of a single dot in interacting environments with diffraction limited spectroscopic methods remains challenging. Therefore, tip-enhanced optical characterizations of OD materials were highly desired in the early stage of nearfield optics. In this section, we discuss tip-enhanced PL measurement of single molecules and quantum dots [103, 106-111]. In addition, we discuss recent observation of PL energy splitting of single quantum dots in the strong coupling regime at room temperature $[58,60]$.

Since the first observation of single molecule signals from a solid crystal at cryogenic temperature by Moerner et al. [112], Betzig et al. demonstrated that a single-molecule detection is even allowed in ambient conditions using illumination-mode NSOM [104]. In that experiment, an apertured fiber probe (diameter of $<100 \mathrm{~nm}$ ) and avalanche photodiode (APD) were used to measure PL from individual carboncyanine dye molecules. These molecules were carefully selected for a photo-stability, and rapid spectral diffusions at room temperature were suppressed by coating the molecules with thin polymethylmethacrylate (PMMA) film. Although this experiment provided many interesting optical properties of single molecules, e. g., non-radiative energy transfer and dipole orientation, luminescence signal through the nano-aperture was obviously too weak to extend its measurement range to broad applications. Therefore, NSOM measurement for single molecules could be performed by only a few research groups [104, 113, 114], and the necessity of higher sensitivity near-field microscopy, such as an ANSOM or a scattering-type NSOM, started blooming. Note that while the ANSOM was not proposed at that time, field-enhancement at the apex of illuminated tip was already studied even earlier than Betzig's singlemolecule NSOM experiment [115].

Several years later, Azoulay et al. presented spectroscopic measurements of single-molecule fluorescence using Si AFM tip [103]. A home-built confocal microscope setup was combined with an AFM to obtain enhanced 
fluorescence from single molecules through localized excitation field at the tip apex. Figure 5 shows measured fluorescence spectra of Rh640 molecules in PVB thin film when the tip is approached (A) to and retracted (B) from the sample. Since they used a non-plasmonic tip for this experiment, the fluorescence enhancement factor was not very large. In addition, the excitation polarization and molecular orientation were not carefully controlled, as well as $30 \mathrm{~nm}$ thick PVB film gave unavoidable limitation in tipsample distance. Notwithstanding, it was the valuable first demonstration of spectral analysis for tip-enhanced singlemolecule fluorescence.

As seen in these early stage works, both apertured and ANSOMs are capable of measuring single molecule fluorescence, yet with low optical sensitivity. To overcome these technical limitations, a number of innovative approaches were presented. For example, Frey et al. showed a hybrid mode of apertured and ANSOM by fabricating metallic tip to an optical fiber probe, so-called tip-on-
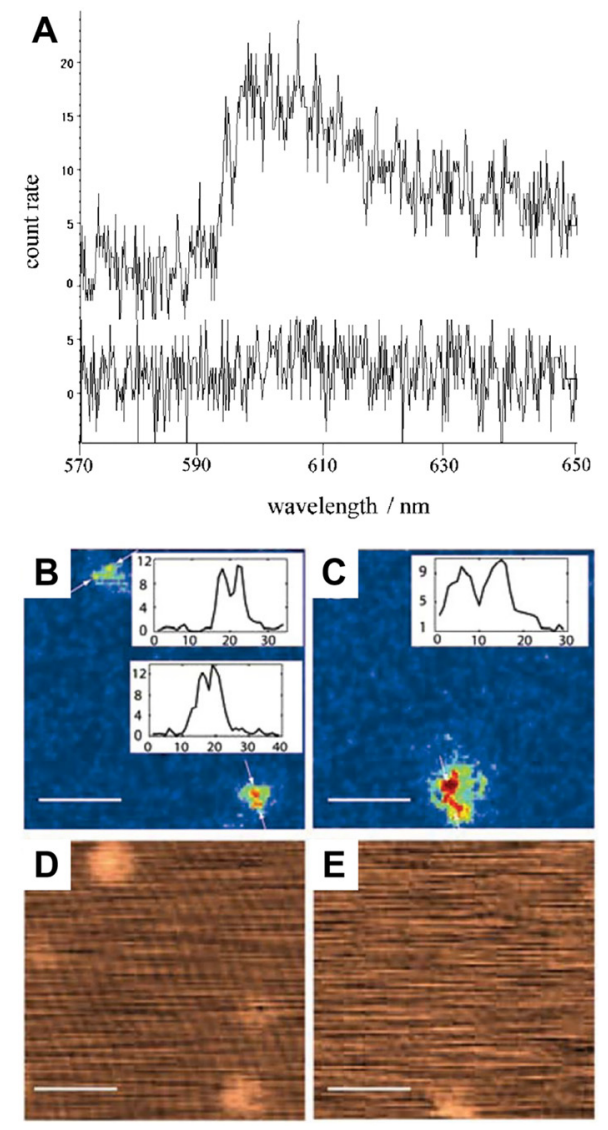

Figure 5: (A) Fluorescence spectra of $\mathrm{Cy} 3$ molecules when Si tip is approached (top) and retracted from sample (bottom). (B-C) Tipinduced fluorescence images of $\mathrm{Cy} 3$ molecules (pair) in different positions. (D-E) Corresponding AFM images of fluorescence images (B) and (C). The scale bars in (B-E) are $50 \mathrm{~nm}$. Reprinted [106] with permission from Copyright 2006 by the American Physical Society. aperture (TOA) NSOM [110]. This approach can improve optical sensitivity and spatial resolution in NSOM imaging via field localization of the metallic tip. Furthermore, farfield background noise, a general problem in apertureless NSOM, is highly suppressed by collecting near-field signals through the fiber-type probe. With this TOA probe, Frey et al. investigated fluorescence properties of single $\mathrm{Cy} 3$ molecules with a spatial resolution of $10 \mathrm{~nm}[110,116]$. Despite these advantages, TOA probe is not widely used due to the complex fabrication process.

While scattering signals in ANSOM are generally stronger than near-field signals through a nano-aperture, the detected scattering light is basically a mixture of nearfield and far-field signals. The unwanted far-field signal is a background noise which can reduce a spatial resolution in near-field imaging, as aforementioned. To solve this problem, Ma et al. developed a novel phase filter to selectively detect near-field fluorescence [106]. In the ANSOM setup with a tapping mode AFM $(\sim 80 \mathrm{kHz})$ and an inverted microscope optics, they characterized the tip-oscillation phase and fluorescence intensity from $\mathrm{Cy} 3$ molecules when the distance between tip and sample is sinusoidally changed. By analyzing correlation between the tip phase and fluorescence intensity, they found a specific phase which gives maximum fluorescence enhancement. Through this phase filter, a signal-to-noise ratio (SNR) was improved $\sim 6$ times for $\mathrm{Cy} 3$ single molecules. In addition, the measured near-field fluorescence image showed a spatial resolution of $8.2 \mathrm{~nm}$, which is even higher resolution than AFM topography, as shown in Figure 5B-E.

Based on these efforts for the advanced instrumentation, the application range of NSOM was extended to biological imaging in aqueous environments with singlemolecule sensitivity. The main difficulty of NSOM operation in liquid is low Q-factor of tuning fork or AFM cantilever. To achieve high Q-factor, Höppener and Novotny used normal-mode AFM based on tuning fork and attached a gold nano-sphere onto the apex of Si tip [108, 117]. This approach enabled to resolve individual $\mathrm{Ca}^{2+}$ pumps and PMCA4 proteins on the cell membrane in PBS solution with $50 \mathrm{~nm}$ spatial resolutions $[108,117]$. The inverse approach of near-field biological imaging in liquid was also demonstrated [33]. This study showed a conventional NSOM probe can be operated in liquid by increasing Qfactor with a novel resonance control method. Further, the single-molecule level sensitivity from membrane proteins could be achieved by labeling plasmonic gold nanoparticles onto the target biomolecules.

The another significant TEPL application for OD materials is a quantum dot. The first experiment of fluorescence enhancement at the Si tip apex was demonstrated by 
Protasenko et al. in 2002 [109]. They prepared ZnS over coated CdSe quantum dots on the prism surface, and excited the sample with a $488 \mathrm{~nm}$, vertically polarized laser to effectively suppress the far-field background with the evanescent illumination. The Si tip was approached to the quantum dots with a tip-sample distance of 5-10 nm in noncontact mode, and the tip-scattered fluorescence signal was measured using a band-pass filter and an APD. The enhancement factor of tip-induced fluorescence was as high as 5-fold for a single isolated quantum dot, and the fluorescence intensity difference for a tip-induced QD and adjacent QD was obtained as high as 90-fold due to the destructive interference between them. This effect also led to high spatial resolution of $30-40 \mathrm{~nm}$ in near-field fluorescence imaging.

In addition, in this early stage study, they found that a range of complex optical processes can occur in the tipsample gap including fluorescence enhancement and quenching dependent on tip preparation condition, materials of tip, and distance between tip and sample. Therefore, TEPL experiment should be carried out carefully, and there are a lot of optical processes to be investigated in the tip-emitter interactions. Note that quenching effect or non-radiative charge transfer to the tip was one significant issue in the early stage of near-field fluorescence spectroscopy and imaging. Traditionally, an intermittent contact mode with an optimized gap between tip and sample was used to minimize this effect with reasonably high fluorescence enhancement $[118,119]$.

In addition to the quenching, another main difficulty of TEPL approach is the far-field background. Since the detected signal is a mixture of far-field and near-field responses, disrupting far-field signal reduces a spatial resolution when the enhancement factor is not high enough. To overcome this limitation, Gerton et al. demonstrated a method to selectively collect near-field signal decoupled from the far-field background by making a large tapping amplitude in intermittent contact mode [111]. When the tip is intermittently contacted to the quantum dots, the near-field excitation rate is maximized as well as near-field PL component. On the contrary, the far-field component out of total detected PL signals is the highest rate when the distance between tip and sample is at maximum. Therefore, by demodulating near-field PL signals with tip oscillating frequency, far-field background can be effectively suppressed. Using this approach, Gerton et al. measured tip-enhanced fluorescence for single isolated CdSe-ZnS core-shell quantum dot, and achieved sub-10 nm spatial resolution by effectively suppressing the far-field signal, as shown in Figure 6A [111]. Figure 6B shows fluorescence intensity as a function of tip-sample distance for three different types of nano-crystals, such as a CdSe-ZnS quantum dot, a CdSe-ZnS nanorod, and a dye-doped latex
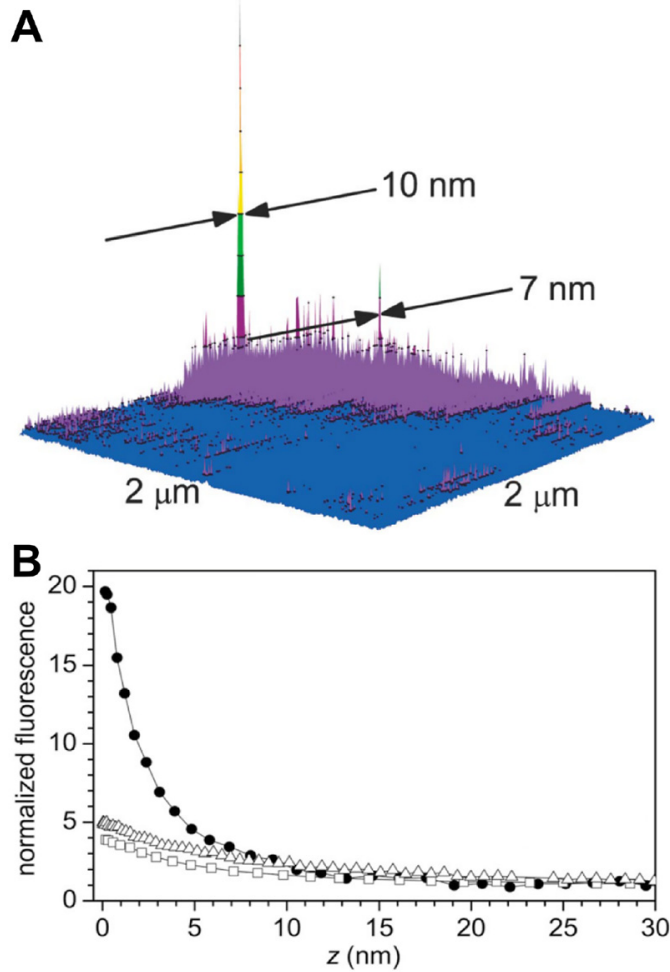

Figure 6: (A) Near-field fluorescence image of $\mathrm{CdSe} / \mathrm{ZnS}$ quantum dots using Si tip. (B) Distance dependent fluorescence intensity between tip and different samples. The distance dependent curves with solid circles, open squares, and open triangles are for a quantum dot of $5 \mathrm{~nm}$ diameter, a nano-rod of $20 \mathrm{~nm}$ length, and a dye-doped latex sphere of $5 \mathrm{~nm}$ diameter, respectively. Reprinted [111] with permission from Copyright 2004 by the American Physical Society.

sphere. This result shows that spatial confinement of nearfield fluorescence regarding tip-induced enhancement is dependent on the size and shape of sample. The halfmaximum of distance dependent TEPL intensity was derived as $1.7 \mathrm{~nm}$ for a quantum dot which is several times smaller value than previous conventional tip-enhanced PL approaches [116, 120-123].

Quantum dots are widely used not only as bright optical sources for modern display devices [124], but also as solid-state single emitters for quantum information devices [125]. Up to present, most TEPL studies of quantum dots were performed in the weak coupling regime, where coupling occurs between exciton and plasmon. Recently, the coupling strength between them was extended to strong coupling regime even at room temperature using TEPL approach $[58,60]$, which provides new insight into dynamic quantum photonic devices. As illustrated in Figure $7 \mathrm{~A}$ and $\mathrm{B}$, the experimental methods are roughly the same as typical TEPL nano-spectroscopy. The key factor to reach the strong coupling regime is the small mode volume $V$ of the tip-cavity because the coupling strength $g$ is 
proportional to $1 / \sqrt{V}$. As seen in Figure $7 \mathrm{~A}$, Groß et al. fabricated slit-like $(<10 \mathrm{~nm})$ plasmonic nano-resonator at a corner of a gold flake, which is mounted at the apex of a tipless AFM cantilever. When single isolated colloidal CdSeTe/ZnS quantum dots are positioned in the small mode volume of this nano-resonator, they could observe energy-split multiple-peaks in the measured TEPL spectra with the coupling strength of up to $110 \mathrm{meV}$. They identified that these results are attributed to collective coupling of a band-edge multiplet of states [58]. A similar but reverse approach was demonstrated by Park et al. using an electrochemically etched gold tip and gold substrate [60]. In the experiment, drop-casted single isolated CdSe/ZnS quantum dots were used as the single emitter. To protect quantum dots from photo-oxidation, gold substrate and quantum dots were coated with a thin $0.5 \mathrm{~nm} \mathrm{Al}_{2} \mathrm{O}_{3}$ capping layer. Using a scanning Au tip operated by a shearforce AFM mode, they could find single quantum dots and control cavity mode volume dynamically. In addition, the tilted tip geometry was used to have maximum field enhancement, as seen in Figure 7B.

Park et al. nicely demonstrated PL spectra of quantum dot excitons, cavity plasmon, as well as TEPL spectra in the weak and strong coupling regime, as shown in Figure 8A. When the cavity loss is larger than the coupling strength, TEPL spectrum is similar to far-field PL of a quantum dot. On the contrary, when the coupling strength exceeds the plasmonic cavity loss, two clear TEPL peak are observed due to the large PL enhancement and Rabi splitting (green). The maximum mode splitting they observed was $\sim 160 \mathrm{meV}$ at room temperature. In addition, from measured TEPL evolution of strongly coupled plexciton, they found that the typical blinking behavior of a quantum dot is also observed in the strong coupling regime (Figure 8B). The main beauty of their works is tuning the coupling strength by controlling the nano-cavity mode volume through the dynamic tip-sample distance control of AFM. Figure 8C shows the transition from strong coupling to weak coupling when Au tip is vertically retracted from a single quantum dot [60]. Prior to these strong coupling works, most tip-enhanced microscopy studies tried to avoid perturbation of sample properties induced by strong plasmon field. On the other hand, these works provide a new paradigm of tip-enhanced microscopy in the deliberate perturbative environments.

\section{Applications to 1D materials}

While 0D quantum materials are important building blocks as an appealing optical source for high performance optoelectronic device applications [60], a separated waveguide structure is needed to transport optical or electronic information of them. In contrast, 1D quantum materials are the most ideal platforms because they not only have strong quantum confinement effect in horizontal axis but also can transfer charges, photons, or other information along the longitudinal axis [126]. Therefore, 1D materials have attracted much attentions as a miniaturized photonic device in the new emerging field of integrated on-chip information technology [127]. To understand optical and electronic properties of 1D materials, a range of PL characterization studies have been performed, yet with diffraction-limited far-field spectroscopy and imaging which cannot provide information on nano-scale heterogeneity [59]. In this section, we discuss TEPL applications on 1D quantum materials which show distinct nano-scale optical properties compared to far-field investigations.

Single-walled carbon nanotubes (SWCNTs) are hollow cylinder shape 1D carbon materials with a diameter of typically smaller than $2 \mathrm{~nm}$ [59]. Semiconducting SWCNTs have low radiative recombination rate and short emission lifetime of photo-excited state due to their unique electronic band structure and dimensional effect [59]. In addition, PL energy of SWCNTs shows inverse proportional behavior with respect to the nanotube diameter [59]. Böhmler et al. demonstrated that these emission properties can be locally controlled at the nano-scale by using a nano-
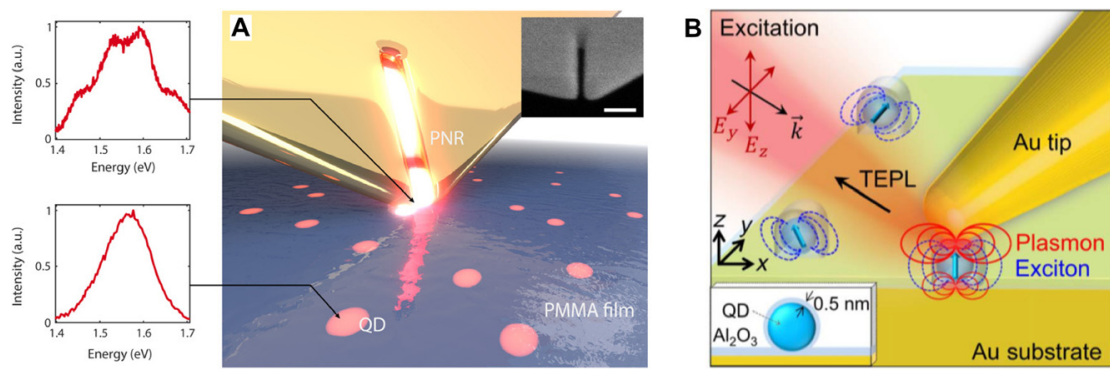

Figure 7: Tip-enhanced photoluminescence spectroscopy experiments for single quantum dots in the strong coupling regime demonstrated by two different groups. (A) Illustration of an experiment with fabricated plasmonic nanoresonator (PNR) and measured far-field and near-field PL spectra of CdSeTe/ ZnS quantum dots. Inset: SEM image of PNR. The scale bar is $100 \mathrm{~nm}$. (B) Illustration of an experiment with a tilted Au tip geometry. From $[58,60]$. Reprinted with permission from AAAS. 

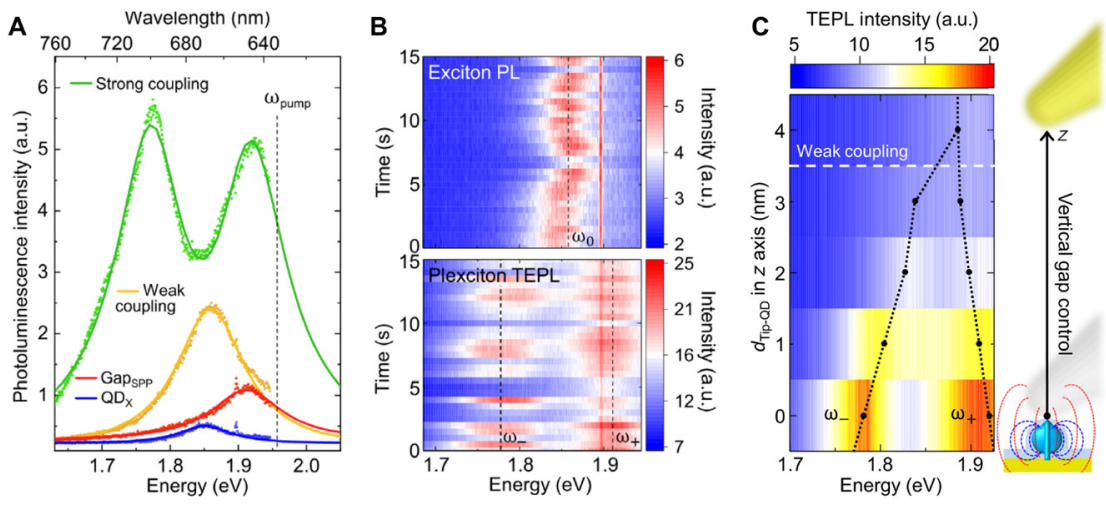

Figure 8: (A) Far-field PL and TEPL spectra of a single $\mathrm{CdSe} / \mathrm{ZnS}$ quantum dot in the weak and strong coupling regimes. Gap plasmon response without a quantum dot is shown with an orange curve. The dots and lines indicate measured data and theoretical model fit, respectively. (B) Time-series of far-field PL spectra of quantum dot excitons (top) and plexciton TEPL spectra for a single quantum dot (bottom). (C) Distance dependent TEPL spectra when the tip is retracted from a single quantum dot from 0 to $4 \mathrm{~nm}$. From [60]. Reprinted with permission from AAAS.

optical antenna-tip [59]. Figure 9A shows a schematic diagram of the measurement setup equipped with an inverted mode microscope and a tuning fork based shear force AFM. The SWCNTs are excited by radially polarized $594 \mathrm{~nm}$ or $633 \mathrm{~nm}$ continuous wave HeNe laser, and the backscattered far-field PL or TEPL signals are detected by an APD and a charge coupled device (CCD). In the detection beam path, a rotating linear polarizer is used to investigate radiation direction of PL (Figure 9B). Since 1D carbon nanotubes can be thought as a chain of point dipole emitters, we expect linearly polarized dipolar emission from the sample. Figure 9C shows simulated radiation patterns for in-plane $\left(\theta=90^{\circ}\right.$, left $)$ and out-of-plane $\left(\theta=0^{\circ}\right.$, right) dipolar emitters. This expected dipolar radiation pattern was observed in polarization resolved far-field PL measurement.

Through external control of excitation conditions with nano-optical antenna-tip, PL properties of 1D material, e. g., radiative emission rate and angular distribution of radiation, can be modified. Figure 9D and E show TEPL image of an SWCNT (inset shows a corresponding AFM topography) and near-field PL radiation pattern when Au tip is located in the arrow indicated in the near-field PL image (D). In contrast to the tip-induced
PL radiation pattern, far-field PL radiation pattern shows linearly polarized behavior as shown in Figure 9F. Figure 9G shows the same result as Figure 9F, but the intensity scale is changed for better comparison with Figure 9E. From these results we know that when Au tip is approached to the SWCNTs, the PL intensity is increased roughly 5 times, as well as emission polarization of single dipole is changed to unpolarized radiation. The enhanced PL intensity is attributed to the increased excitation and radiative emission rates in the vicinity of Au tip. The observed circular radiation pattern is typical characteristic of a point dipole oriented vertically. Therefore, this result demonstrates that when the tip is closed to the SWCNT, a new radiation channel is created and the radiation energy is transferred to the tip. In this case, the scattered PL emission with the ring-shape radiation pattern is detected [59]. This work demonstrates that heterogeneous optical and electronic properties of 1D materials can be resolved with TEPL imaging. Additionally, PL properties of 1D emitters are allowed to control with improved performances at the nano-scale through nano-optical antenna-tip.

In addition to the modified dipolar emission of the SWCNTs in near-field [59], understanding their spatial
A

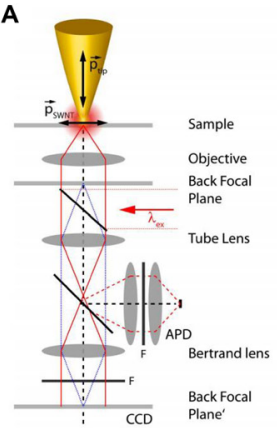

B

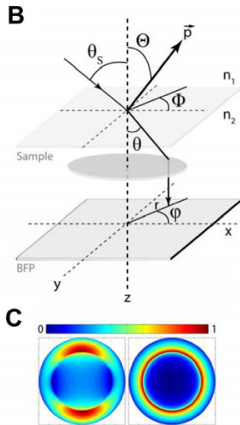

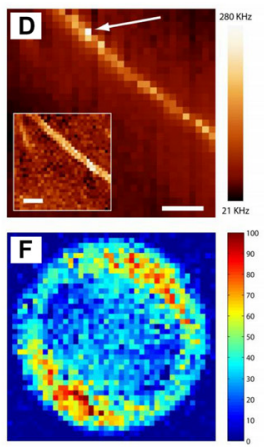

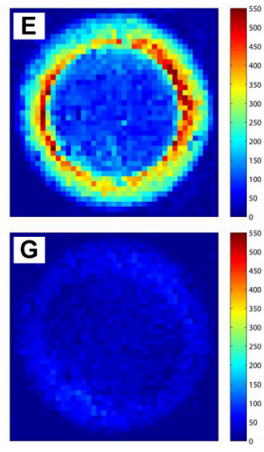

Figure 9: (A) Schematic diagram of near-field PL measurement setup. (B) Modeling of the detection beam path to analyze $P L$ radiation pattern. (C) Simulated radiation patterns for the two different point dipole conditions, i. e., horizontally oriented $\left(\theta=90^{\circ}\right)$ and vertically oriented $\left(\theta=0^{\circ}\right)$ dipoles. (D) TEPL image and topography of the SWCNT. The scale bar is $100 \mathrm{~nm}$. Tip-enhanced nearfield $(\mathrm{E})$ and far-field $(\mathrm{F}, \mathrm{G}) \mathrm{PL}$ radiation patterns at the point indicated by an arrow in TEPL image (D). (G) is the same image as (F), but the intensity scale is modified for 
heterogeneity in optical and electronic properties is of significance to material physicists. Qian et al. visualized the nanoscale optical heterogeneity of semiconducting carbon nanotubes wrapped by DNA with spectral analysis [128]. The helical wrapping of a carbon nanotube by the charged DNA backbone induces $\pi-\pi$ stacking interactions between them, as well as electrostatic interactions [129131]. These effects in the DNA-nanotube hybrid structure make change in relative dielectric constant of the nanotube, which leads to modifications in electronic properties, such as exciton binding energy and band gap. Additionally, it facilitates symmetry breaking in the electronic band structure of carbon nanotubes which gives rise to PL energy shifts. To characterize these modified local optical responses, TEPL nano-imaging study was performed [128]. As sample systems, DNAwrapped nanotubes were spin-coated on a thin mica layer/cover slip. To adhere, the naturally negatively charged DNA onto the surface, the mica layer was made to have positive charges with $\mathrm{Mg}^{2+}$ ions [129]. TEPL setup was based on an inverted optical microscope with confocal PL optics, and a distance between a sharp metallic tip and the sample was regulated by a shear-force feedback mechanism. With the highly improved spatial resolution of TEPL compared to confocal PL imaging, DNA-induced PL energy transition of carbon nanotube was visualized at the nano-scale [128]. Figure 10A and B show AFM topography of a DNA-wrapped carbon nanotube and corresponding TEPL image. To visualize the TEPL image, the integrated PL intensity between 970 and 1,030 nm, i. e., corresponding to emission range of the sample, was used. Figure 10C and D shows position-dependent peak emission energies along the $1 \mathrm{D}$ nanotube structure derived from the measured TEPL spectra. An obvious spectral fluctuation between 1.241 and $1.259 \mathrm{eV}$ was observed due to the non-uniform DNA-wrapping around the nanotube. As can be seen in Figure 10D, the length scale in the transition of PL peak energy is sub-diffraction limit. Therefore, the observed nano-scale heterogeneity could not be resolved with confocal PL imaging. As a matter of fact, the transition between PL energy levels of DNAwrapped and unwrapped segments occurs on a smaller length scale than the spatial resolution of TEPL imaging $(15 \mathrm{~nm})$. Distance dependent intensity profiles (Figure 10E) and individual TEPL intensity maps for the $1.259 \mathrm{eV}$ (unwrapped) and $1.259 \mathrm{eV}$ (wrapped) peaks in Figure 10F and $\mathrm{G}$ shows a clear anti-correlated behavior. Note that, from the tip-sample distance dependence of TEPL measurement, they confirmed that the metallic tip does not perturb the PL emission energy of the sample [128].
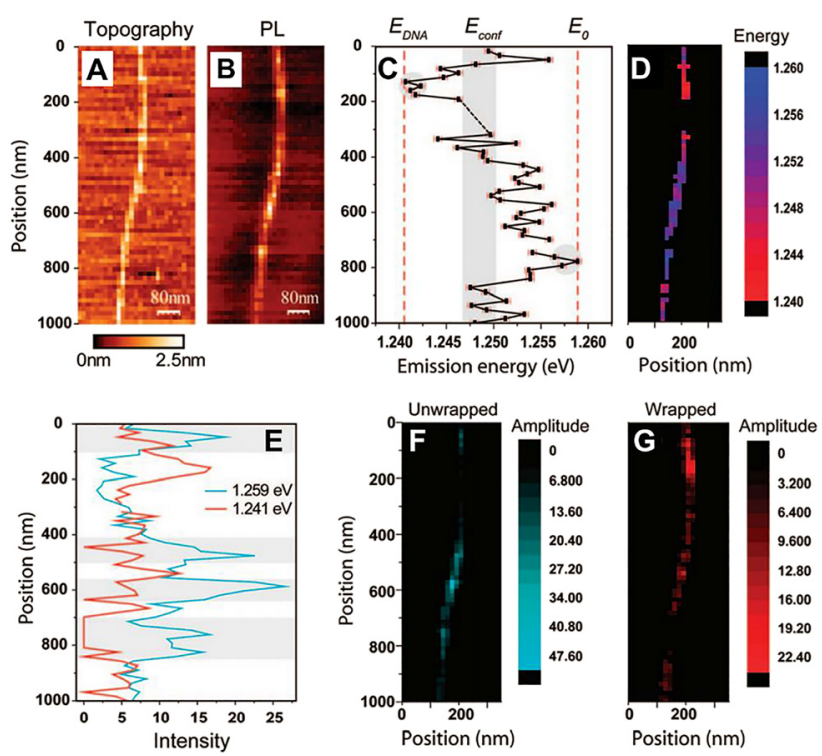

Figure 10: AFM topography (A) and simultaneously measured nearfield PL image (B) of a DNA-wrapped carbon nanotube on the mica substrate. (C, D) Position-dependent peak emission energies derived from the TEPL spectra along the 1D sample. To derive the peak values at each position, the measured spectra were fitted with a Lorentzian line function. (E) Position-dependent PL intensities for emission peaks of $1.241 \mathrm{eV}$ (DNA-wrapped segments) and $1.259 \mathrm{eV}$ (DNA-unwrapped segments). TEPL intensities with respect to the position of a carbon nanotube for the peaks of $1.259 \mathrm{eV}(\mathrm{F})$ and $1.241 \mathrm{eV}(\mathrm{G})$ [128]. Reprinted with permission from [128]. Copyright (2008) American Chemical Society.

In addition to the carbon nanotubes, CdSe nanowire is also one of the significant 1D material for integrated photonic devices, photo-voltaics, and sensing devices owing to their strong photo-induced responses and controllability [132]. For example, CdSe nanowires have much higher absorption cross-section compared to CdSe quantum dots. In addition, the quantum confinement of CdSe nanowires is highly increased when the diameter of nanowires is less than $11 \mathrm{~nm}$ because CdSe has an exciton Bohr radius of $5.6 \mathrm{~nm}$ and the electronic band gap energy is inverse proportional to the square of a diameter due to the changed quantum confinement effect [126].

In 2011, Böhmler et al. demonstrated tip-enhanced PL and Raman characterizations of CdSe quantum wires and revealed the diameter-dependent quantum confinement effect [132]. Figure 11A shows a brief schematic diagram of the experimental setup, which consists of a confocal microscope and a shear-force AFM. CdSe nanowires (Figure 11B) were dispersed on a glass substrate and excited by a $632.8 \mathrm{~nm}$ radially polarized HeNe laser. An electrochemically etched $\mathrm{Au}$ tip was used to scatter nearfield PL signal of the sample, and the signal was detected either by an APD with spectral filtering or a CCD with a 
spectrometer. Strong field enhancement at the tip apex leads to significant improvements in both spatial resolution and PL intensity compared to confocal PL measurement. Figure $11 \mathrm{C}$ shows a topography of CdSe quantum wires measured with tip-enhanced near-field optical microscopy and Figure 11D and E shows simultaneously measured near-field PL and Raman images. With the improved spatial resolution of $20 \mathrm{~nm}$ in near-field images, the diameter difference of two wire branches (I and II) and their tripod structure is clearly revealed. Additionally, spectral differences in PL between thin (II) and thick (I) nanowires were visualized which could not be resolved with the conventional far field PL imaging. The PL energy shift between thin and thick wires was observed up to several tens of meV. Figure 11F shows near-field PL spectra measured at branches I and II. The thicker wire (I) gives PL response at lower energy (red) compared to the thinner wire (II, blue) due to the decreased band gap. As can be seen in Figure 11E, stronger Raman scattering signal was obtained from the thinner quantum wire due to the resonance Raman effect [132].

\section{Applications to 2D materials}

Transition metal dichalcogenide monolayers are emerging 2D semiconductors which have unique excitonic properties. Dimensional reduction of TMDs from bulk to monolayer gives rise to direct band-to-band transition of electric charges, e. g., an exfoliated $\mathrm{MoS}_{2}$ monolayer shows direct bandgap at $\sim 1.8 \mathrm{eV}$ with strong PL emission even though its bulk state (multilayers) has an indirect bandgap at $\sim 1.3 \mathrm{eV}$ $[133,134]$. In addition, stable neutral excitons, biexcitons, and trions are formed in 2D TMDs even at room temperature with large exciton binding energy due to the strong Coulomb interaction, whereas optical and electronic properties of bulk state are determined by free charge carriers [135, 136]. Therefore, TMD monolayers have attracted much attentions as 2D semiconductors and, controlling these unique excitonic properties have potential applications for the next generation ultra-thin, -light, and flexible optoelectronic devices [137]. For practical applications of TMD-based optoelectronic device, characterization of optical and electronic properties should be preceded. These properties on the microscopic scale can be easily understood from spectroscopic far-field PL measurement [138, 139]. However, nano-scale optical and electronic properties at the defects of 2D TMD, such as impurities, vacancies, grain boundaries, and edges cannot be understood with a conventional far-field PL spectroscopy due to the diffraction limit. Therefore, TEPL characterization of these nano-scale defects and spatial heterogeneity is highly desirable. In addition, tip-induced local control of TMD excitonic properties, e. g., PL enhancement and bandgap modification, provides a new point of view for novel device platforms [140]. In this section, we discuss recent progress of TEPL applications to 2D TMD materials.

Park et al. demonstrated TEPL nano-spectroscopy and -imaging for a WSe 2 monolayer in 2016 [20]. This work was the first TEPL approach for 2D TMDs with high sensitivity and spatial resolution, even though a few apertured NSOM works were performed prior to this work [141, 142]. The experiment is basically based on a conventional confocal microscope setup which uses illumination from the top with atomic force microscope (AFM) tip for performing TERS and TEPL. Helium-Neon laser $(632.8 \mathrm{~nm},<0.5 \mathrm{~mW})$ is focused through the objective lens $(100 \times, \mathrm{NA}=0.8)$ and the electrochemically etched gold tip with apex radius $(<15 \mathrm{~nm})$ is fixed in the center of focal area. The tip is tilted with $\sim 40^{\circ}$ with respect to the vertical line of the sample surface [20]. Figure 12A shows confocal PL (top left) and TEPL images
A
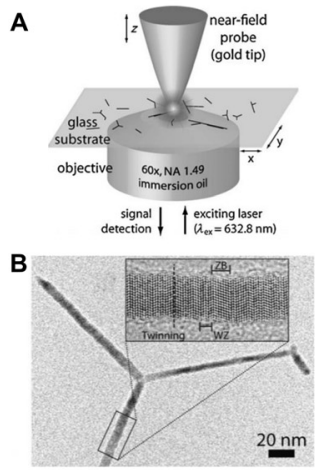

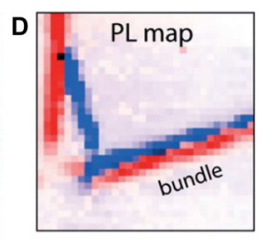

$\mathbf{F}$
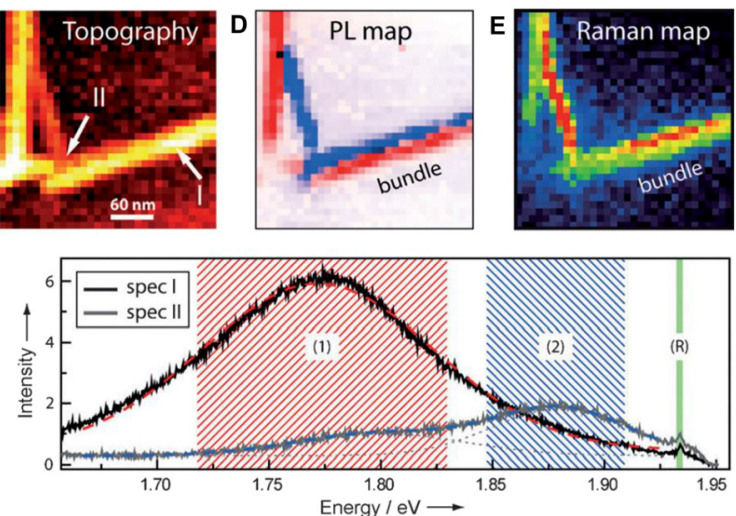

Figure 11: (A) Schematic diagram of tipenhanced near-field optical microscopy setup. (B) Transmission electron microscopy (TEM) image of CdSe quantum wires. (C) Topography of branched CdSe nanowire measured with shear-force AFM. (D-E) Corresponding near-field intensity maps of PL and Raman responses. (F) Nearfield PL spectra at the positions I and II indicated in (C) [132]. Copyright 2011 by John Wiley Sons, Inc. Reprinted by permission of John Wiley \& Sons, Inc. 
(bottom) of a chemical vapor deposition (CVD) grown $\mathrm{WSe}_{2}$ monolayer on $\mathrm{SiO}_{2}$ substrate with corresponding AFM topography. Compared to confocal PL image, TEPL images show clear nano-scale defects, such as twin boundary, nucleation site, and edge, with highly enhanced PL intensity and spatial resolution $<15 \mathrm{~nm}$. In addition, an obvious change in PL intensity with spectral peak shift is observed in the spectral TEPL analysis as shown in Figure 12B. Figure 12C shows TEPL spectra and deconvolution of exciton and biexciton peaks obtained from the crystal edge, nucleation site, and crystal face. Compared to the PL response at the crystal face, the decreased PL intensity with slight spectral blue-shift at the edge is attributed to energy funneling from higher energy states to lower ones in the heterogeneous and disordered semiconducting system. This PL modification at the edge is observed over $\sim 30-80 \mathrm{~nm}$ spatial extent, which is a similar length scale to the edge roughness of TMD crystal [143]. The distinct blue-shift with largely decreased PL intensity at the nucleation site is possibly due to the imperfect composite ratio of Se [144], as well as W residue on the surface of the crystal which can be a channel of non-radiative recombination [145]. Furthermore, through the high spatial resolution TEPL measurement and spectral analysis at twin boundaries, the exciton diffusion length of $\sim 25 \mathrm{~nm}$ at room temperature is directly quantified. This work demonstrated that high resolution TEPL nano-spectroscopy and -imaging allows to reveal heterogeneous optical and electronic properties at the nanoscale defects of $2 \mathrm{D}$ TMD crystals.

Since understanding local excitonic processes was desirable at the early stage of new emerging TMD monolayers, similar TEPL work has done by Su et al. for $\mathrm{MoS}_{2}$ monolayer at about the same time [55]. They performed direct nano-scale PL mapping of excitons and trions and found the difference in PL enhancement factor between them due to the influence of the metal-tip work function. For the experiment, an exfoliated $\mathrm{MoS}_{2}$ monolayer was prepared on a glass coverslip, and a radially polarized
$532 \mathrm{~nm}$ laser was focused on the sample surface using an inverted microscope. Ag- or Au-coated Si cantilevers were used for excitation field localization and PL enhancement. The distance regulation between tip and sample and sample scanning were carried out using contact-mode commercial AFM. Figure 13A shows Raman and PL spectra of $\mathrm{MoS}_{2}$ monolayer with and without the Ag-coated tip. When the tip is approached onto the sample, Raman and trion PL intensities are enhanced overall, whereas PL intensity of $\mathrm{A}_{0}$ exciton is decreased. The enhanced Raman and trion PL signals are explained by the highly enhanced excitation rate at the tip apex because absorption resonance of the Ag-coated tip ( $2.4 \mathrm{eV})$ is close to the excitation beam energy $(2.3 \mathrm{eV})$. On the other hand, the observed decrease in $\mathrm{A}_{0}$ exciton PL is caused by the work function of $\mathrm{Ag}(4.3 \mathrm{eV})$. Because the work function of $\mathrm{Ag}$ is lower than the work function of monolayer of $\mathrm{MoS}_{2}(4.7 \mathrm{eV})$, electrons of Ag tip naturally move to the $\mathrm{MoS}_{2}$ crystal which causes ntype doping effect and PL decrease. As for the comparison with the Ag-coated tip, when the Au-coated tip is approached to the same sample, $A_{0}$ exciton PL signal is enhanced, yet Raman scattering intensity stays the same, as shown in Figure 13B. This result shows that there is no significant enhancement of the excitation field because the plasmon resonance of Au-coated tip (1.9 eV) is quite far from excitation energy. Therefore, the enhanced PL of $A_{0}$ exciton is mainly attributed to the charge transfer effect rather than the increased excitation rate. Figure 13C shows energy diagram of $\mathrm{MoS}_{2}$ and metallic tips which explains the flow of electrons and p- or n-doping effect of TMD crystals depend on the material of tip. The unchanged PL intensity of trions with the Au tip is also explained with this p-doping effect. Figure 13D shows an overlay image of TERS intensity for $\mathrm{A}_{1 \mathrm{~g}}$ mode and integrated TEPL intensity for A-exciton band using the Ag-coated tip. Figure 13E and $\mathrm{F}$ shows TEPL maps of $\mathrm{A}_{0}$-excitons and trions obtained from deconvolution of Figure 13D. For comparison, TEPL
A
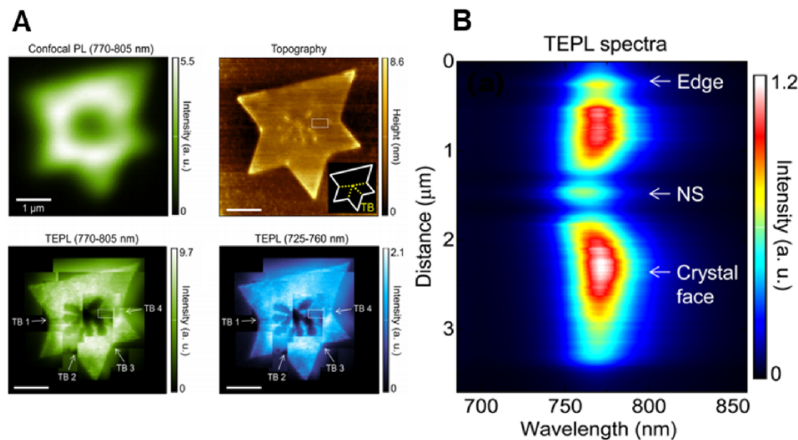

Figure 12: (A) PL images of a WSe $\mathrm{W}_{2}$ monolayer measured with confocal microscopy (top) and TEPL microscopy (bottom, the integrated intensity of $770-805 \mathrm{~nm}$ and $725-760 \mathrm{~nm}$ ) with the corresponding AFM topography. (B) TEPL spectra along the center axis of a WSe $\mathrm{W}_{2}$ monolayer. (C) TEPL spectra with peak energies of $1.610 \mathrm{eV}$ at the edge, $1.630 \mathrm{eV}$ at the nuclear site (NS), and $1.606 \mathrm{eV}$ at the crystal face. Exciton (blue) and biexciton (red) peaks are fitted with Voigt line function. Reprinted with permission from [20]. Copyright (2016) American Chemical Society. 

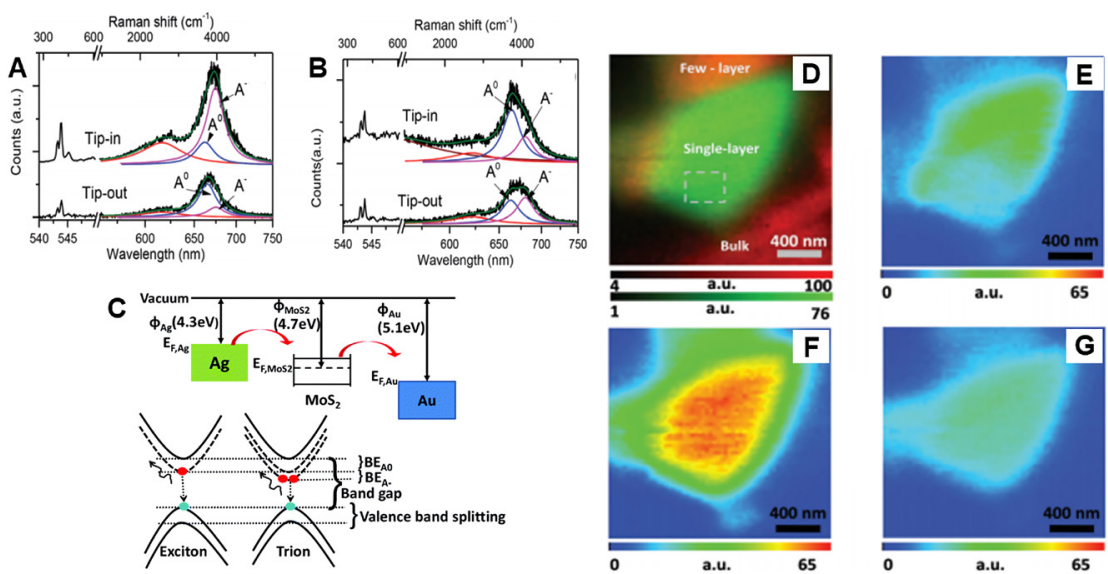

Figure 13: $(A-B) P L$ and Raman spectra of a $\mathrm{MoS}_{2}$ monolayer when a metallic tip is approached and retracted. Ag-coated tip (A) and $A u$-coated tip (B) were used for these measurements. Blue, purple, and red lines represent the $P L$ intensity of $A^{\circ}, A^{-}$, and $B$ excitons, respectively. (C) Energy diagram exhibiting work functions of $\mathrm{MoS}_{2}, \mathrm{Ag}$, and Au with the flow of electric charges. (D) Overlay near-field image of TERS (red) and TEPL (green) signals of $\mathrm{MoS}_{2}$ monolayer. TEPL images of $A^{0}$ excitons (E) and trions ( $F$ ), and $\mathrm{B}$-excitons $(\mathrm{G})$ obtained by deconvoluting the measured near-field $\mathrm{PL}$ spectra. Reproduced from [55] with permission from The Royal Society of Chemistry.

map of B-excitons is shown in Figure 13G. In comparison with the far-field measurement, PL intensity of trions and B-exciton are highly enhanced, yet PL quenching behavior is shown for $\mathrm{A}_{0}$-exciton with TEPL. In addition, far-field PL intensity is dominated by the emission of $\mathrm{A}_{0}$-exciton, but near-field PL intensity is mainly contributed by trion emission. This study nicely demonstrated the local distributions of excitonic components, such as excitons and trions, at the nano-scale with the tip-induced effects.

Similar TEPL and TERS works for TMD monolayer were carried out by Okuno et al. [146]. They also demonstrated the nano-scale heterogeneity in optical properties for a CVD grown $\mathrm{MoS}_{2}$. Furthermore, they showed that the local variations PL properties are associated with the chemical environments. Figure 14A shows TEPL image of short $\mathrm{MoS}_{2}$ flake. The dark spot on the center is due to the presence of a nanoscale terrace, which was also confirmed with the AFM topography. Although the observed nano-scale terrace was indeed confirmed as a multilayer structure, the $\mathrm{MoS}_{2}$ flake is expected to be a monolayer because only PL intensity is decreased with no peak shift. The other sample was prepared to have both single- and bi-layer flakes on the substrate. Figure 14B and D shows TEPL image and AFM topography measured simultaneously. As expected from the difference in electronic band structure of single- and bilayer TMDs, strong PL emission was measured only from monolayer flakes. Additionally, stronger TERS signal was observed from single layer flakes, as shown in Figure 14C. In their experiment, bilayer structure was confirmed by the Raman peak shift of $A_{1 g}$ mode as well as topographic line profile (Figure 14E).

Beyond the TEPL imaging of TMD crystals to understand intrinsic optical and electronic properties, control of these properties of TMDs through the nano-opto-mechanical tip force control was presented by Park et al. [20]. For the experiment, $\mathrm{WSe}_{2}$ monolayers grown on $\mathrm{SiO}_{2} / \mathrm{Si}$ substrate was used. The as-grown crystal is under a tensile strain of $\sim 1 \%$ through physical vapor deposition. To release the initially applied tensile strain of the crystal, etched $\mathrm{Au}$ tip was used. By carefully applying the tip contact force
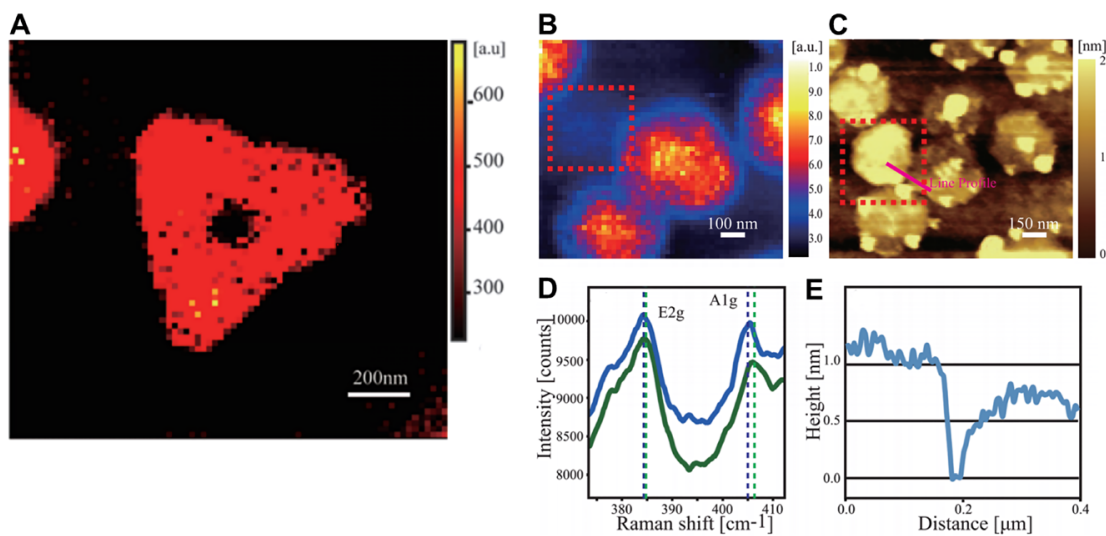

Figure 14: (A) Spectrally integrated TEPL image $\mathrm{MoS}_{2}$ nano-flake. TEPL image (B) and AFM topography (C) of single- and bilayered $\mathrm{MoS}_{2}$ nano-flakes. (D) TERS spectra of $\mathrm{MoS}_{2}$ monolayer (blue) and bilayer (green). (E) A topographic line profile of the sample indicated in (C). Reproduced from [146] with permission from The Royal Society of Chemistry. 

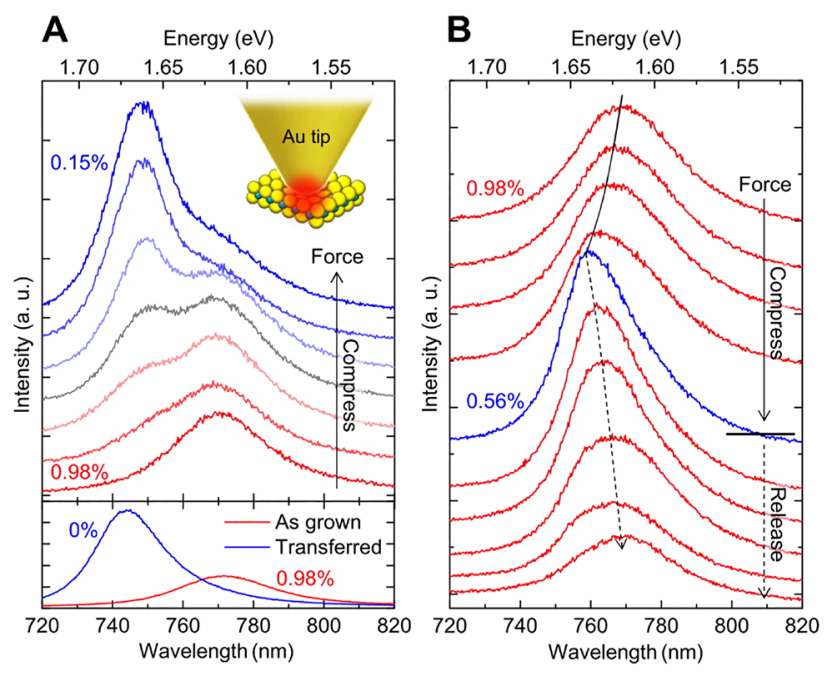

Figure 15: (A) Evolution of TEPL spectra of a WSe $e_{2}$ monolayer under the tip-force regulation exhibiting irreversible PL energy shift (top). Far-field PL spectra of as-grown and transferred $\mathrm{WSe}_{2}$ monolayer on the $\mathrm{SiO}_{2}$ substrate (bottom). (B) Evolution of TEPL spectra of a WSe monolayer under weaker tip interaction exhibiting reversible $\mathrm{PL}$ energy shift. Reprinted with permission from [20]. Copyright (2016) American Chemical Society.

onto the local spot and retracting the tip from the crystal, the tensile strain could be released at the nano-scale. They could monitor the changed strain by measuring TEPL spectra simultaneously. Figure 15A shows an irreversible strain shift with respect to the increased compressive force by the tip regulation. The induced tensile strain on the crystal was estimated from the peak shift of simultaneously measured TEPL spectra. The maximum $48 \mathrm{meV}$ of PL peak shift was observed through the nano-mechanical tip-force regulation. Note that PL quantum yield of TMD crystal is decreased under strain due to the modified electronic band structure [147]. Furthermore, with the weaker tip-force control, tensile strain of the crystal and corresponding TEPL enegry could be reversibly controlled, as shown in
Figure 15B. Controlling optical and electronic properties of semiconductors is essential for device applications. Conventional methods, such as controlling impurities, defects, and external dopants, are widely used, yet it is difficult to control at the nano-scale region [148]. This work presented a novel approach to dynamically control strain, band gap, and PL energy of atomically thin semiconductor at the nano-scale.

Recently, He et al. demonstrated the local control of radiative emission of neutral excitons and trions in $\mathrm{WS}_{2}$ monolayer through precision gap control between $\mathrm{Ag}$ tip and $\mathrm{Au}$ substrate [149]. Figure $16 \mathrm{~A}$ shows a schematic illustration of the experimental setup. They used $\mathrm{Au}$ coated Ag tip which was mounted on a cantilever. The distance between the tip and substrate was regulated with contact-mode AFM and precisely calibrated using the force-distance analysis [55]. Figure 16B shows an illustration of the classical coupling regime of TEPL spectroscopy. In this coupling regime, the tip-sample distance is $\sim 1 \mathrm{~nm}$ and PL responses for neutral exciton $\left(\mathrm{X}_{0}\right)$ and trions $\left(\mathrm{X}^{-}\right)$are observed, as shown in Figure 16C. In contrast, when the gap is decreased to $\sim 0.3 \mathrm{~nm}$, direct tunneling of electrons from Ag tip to $\mathrm{WS}_{2}$ is induced. In this regime, these tunneling charges facilitate the transition of neutral excitons to trions, as described in Figure 16D. Therefore, the measured TEPL spectra under this regime show highly increased trion emission, as shown in Figure 16E. Further, as seen in Figure 16F, when the tip-sample distance becomes smaller than $250 \mathrm{~nm}$, PL intensity of trions increases in contrast to the decreased PL intensity of neutral excions. This result shows an obvious evidence of neutral exciton-trions conversion with respect to the picometer gap between tip and sample. This work demonstrated the quantum plasmonic effects on $\mathrm{WS}_{2}$ monolayer in sub- $1 \mathrm{~nm}$ gap of metallic cavity which opens new regimes of nano-scale control of TMD excitonic processes.

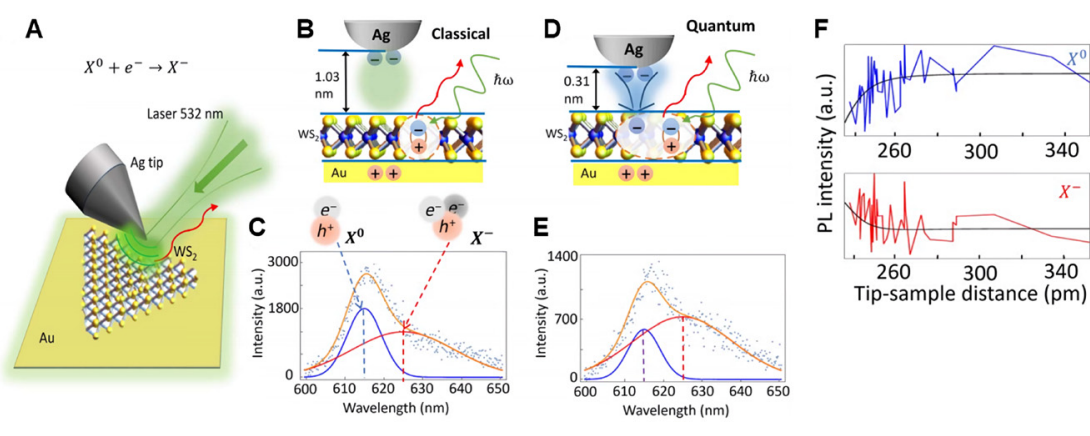




\section{Conclusions and outlook}

Since Synge's old proposal to overcome diffraction limit in far-field optical imaging [24], near-field scanning microscopy has advanced considerably with the development of SPM technology in 1980s [150-152]. The initial approach to implement near-field microscopy is NSOM using a metalcoated optical fiber probe $[25,26]$. NSOM is an innovative nano-imaging technique with a spatial resolution of $50 \sim 100 \mathrm{~nm}$, but several inevitable difficulties in using aperture-type probe, especially weak optical signals, have restricted the popularization and broad applications. An inverse approach inducing near-field scattering by the tip, e. g., $s$-SNOM, made improvements in near-field microscopy by solving many problems of NSOM with an improved spatial resolution of $<10 \mathrm{~nm}[29,40-44]$. However, obtaining high spatial resolution image is still challenging due to the far-field background. To this end, tip-enhanced nano-imaging approach has been developed using the LSPR effect of plasmonic tip [47-49]. With combined nano-spectroscopic measurement of Raman and PL, i. e., TERS and TEPL, its application range is widely extended. Recently, TEPL approach further advanced to the strong coupling regime using plasmonic tip-cavity, i. e., TESC spectroscopy [60].

We focused on the principles and applications of TEPL in this review. Basically, the enhancement factor of TEPL signal is determined by the tip-induced excitation rate and the spontaneous emission rate [69]. Since the spontaneous emission rate is increased by the Purcell effect, we can achieve larger enhancement using metal substrates by making smaller mode volume, which also enables large excitation rate based on the coupled dipole model [37, 63, 64]. To demonstrate application studies, we discussed TEPL results on the low-dimensional quantum materials. For the sake of convenience, we classified the results by the dimension, i. e., $0 \mathrm{D}, 1 \mathrm{D}$, and $2 \mathrm{D}$ materials. In the $\mathrm{OD}$ application section, we discussed TEPL studies of single molecules and single quantum dots from weak to strong coupling regimes. We then discussed TEPL applications on 1D materials, such as single-walled carbon nanotubes and CdSe quantum wires. Lastly, we presented recent TEPL nano-spectroscopy and nano-imaging results on 2D TMDs.

In addition to the materials we have discussed in this review, many kinds of new emerging low dimensional material systems are being studied today. Therefore, TEPL will be a more important characterization method to understand optical and electronic properties of new emerging materials at the nano-scale. Beyond the high resolution nano-spectroscopy, this tip-enhanced PL approach can be used to dynamically control quantum light-matter interactions at the nano-scale. If we use highly conductive metal substrates, the field enhancement and mode volume of the tip-cavity are dramatically changed depending on the tip-substrate gap. By precisely regulating tip-substrate distance with atomic force feedback, we can control the plasmon strength, which is easily coupled to other quasiparticles of materials, such as excitons. Recently, Park et al. demonstrated several studies on the tip-induced control of material properties, e. g., reversible control of PL energy for TMD monolayers and single quantum dots in the weak and strong coupling regimes [60]. As for the perspective of instrumentation, currently most TEPL studies were performed in ambient conditions. For broader applications of TEPL approach as well as groundbreaking works, the advanced instrumentation studies must take precedence. For example, low-temperature TEPL setup will allow to measure intrinsic excitonic properties with narrower PL linewidth and higher intensity by suppressing thermally activated processes, e. g., relaxation, quenching, and photoionization $[153,154]$. This implementation enables to measure atomic defects of low-dimensional quantum materials in great detail. In addition, cryogenic temperature will be more favorable for TESC experiment because optical losses in plasmonic tip and substrates are decreased. For application to soft and living matters, such as biological samples, a routine operation of TEPL setup in liquid is desirable even though it has remained challenging. Furthermore, TEPL measurement for device systems with in situ control of electrical and electronic properties will be a valuable advance. Because this multifunctional measurement will allow to understand correlation information of structural, optical, electronic, and electrical properties. The other challenge is near-field polarization control at the apex of plasmonic tip [57]. As we discussed in section 4, the polarizability and the field intensity are dominant in the axis perpendicular with respect to the sample surface. On the other hand, each of the materials has a different orientation of transition dipole moment, e. g., the electric dipole orientation of $1 \mathrm{D}$ and $2 \mathrm{D}$ materials is preferably into in-plane direction $[59,20]$. Therefore, controlling near-field polarization of in-plane and out-of-plane components will be an innovative approach for effective TEPL measurement as well as nanocrystallography applications.

Acknowledgment: This work was supported by the National Research Foundation of Korea (NRF) grant funded by the Korea government (MEST) (No. 2019R1F1A1059892, 2019K2A9A1A06099937, and 2020R1C1C1011301). This work was supported by the Start-Up Research Fund 
(1.180091.01) of UNIST (Ulsan National Institute of Science \& Technology).

\section{References}

[1] L. J. A. Koster, E. C. P. Smits, V. D. Mihailetchi, and P. W. M. Blom, "Device model for the operation of polymer/fullerene bulk heterojunction solar cells.," Phys. Rev. B, vol. 72, no. 8, 2005, Art no. 085205 , https://doi.org/10.1103/physrevb.72.085205.

[2] G. K. Mercouri, Semiconductors and semimetals, Amsterdam, Netherlands, Elsevier, 2001.

[3] J. Li, A. Corma, and J. Yu, "Synthesis of new zeolite structures.," Chem. Soc. Rev., vol. 44, no. 20, pp. 7112-7127, 2015, https:// doi.org/10.1039/c5cs00023h.

[4] T. Takagahara and K. Takeda, "Theory of the quantum confinement effect on excitons in quantum dots of indirect-gap materials.," Phys. Rev. B, vol. 46, no. 23, p. 15578, 1992, https:// doi.org/10.1103/physrevb.46.15578.

[5] S. Latini, T. Olsen, and K. S. Thygesen, "Excitons in Van der Waals heterostructures: The important role of dielectric screening.," Phys. Rev. B, vol. 92, no. 24, p. 245123, 2015, https://doi.org/10.1103/physrevb.92.245123.

[6] Z. B. Sheneve, M. H. Shawna, L. Cao, et al., "Progress, challenges, and opportunities in two-dimensional materials beyond graphene.," ACS Nano, vol. 7, no. 4, pp. 2898-2926, 2013, https://doi.org/10.1021/nn400280c.

[7] A. K. Geim, “Graphene: Status and prospects.," Science, vol. 324, no. 5934, pp. 1530-1534, 2009, https://doi.org/10.1126/ science. 1158877.

[8] M. S. Dresselhaus, G. Chen, M. Y. Tang, et al., "New directions for low-dimensional thermoelectric materials.," Adv. Mater., vol. 19, no. 8, pp. 1043-1053, 2007, https://doi.org/10.1002/adma. 200600527.

[9] A. C. Ferrari, J. C. Meyer, V. Scardaci, et al., "Raman spectrum of graphene and graphene layers.," Phys. Rev. Lett., vol. 97, no. 18, p. 187401, 2006, https://doi.org/10.1103/physrevlett.97. 187401.

[10] T. W. Odom, J. L. Huang, P. Kim, and C. M. Lieber, “Atomic structure and electronic properties of single-walled carbon nanotubes.," Nature, vol. 391, no. 6662, pp. 62-64, 1998, https://doi.org/10.1038/34145.

[11] R. Addou, L. Colombo, and R. M. Wallace, "Surface defects on natural $\mathrm{MoS}_{2}$.," ACS Appl. Mater. Interfaces, vol. 7, no. 22, pp. 11921-11929, 2015, https://doi.org/10.1021/acsami. 5 b01778.

[12] H. Kirmse, R. Schneider, M. Rabe, W. Neumann, and F. Henneberger, "Transmission electron microscopy investigation of structural properties of self-assembled CdSe/ZnSe quantum dots.," Appl. Phys. Lett., vol. 72, no. 11, pp. 1329-1331, 1998, https://doi.org/10.1063/1.120984.

[13] H. J. Liu, L. Jiao, L. Xie, et al., “Molecular-beam epitaxy of monolayer and bilayer $\mathrm{WSe}_{2}$ : A scanning tunneling microscopy/ spectroscopy study and deduction of exciton binding energy.," 2D Mater., vol. 2, no. 3, p. 034004, 2015, https://doi.org/10. 1088/2053-1583/2/3/034004.

[14] K. Xu, P. Cao, and J. R. Heath, "Scanning tunneling microscopy characterization of the electrical properties of wrinkles in exfoliated graphene monolayers.," Nano Lett., vol. 9, no. 12, pp. 4446-4451, 2009, https://doi.org/10.1021/nl902729p.

[15] S. Loth, M. Etzkorn, C. P. Lutz, D. M. Eigler, and A. J. Heinrich, "Measurement of fast electron spin relaxation times with atomic resolution.," Science, vol. 329, no. 5999, pp. 1628-1630, 2010, https://doi.org/10.1126/science.1191688.

[16] S. Yoshida, Y. Aizawa, Z. Wang, et al., "Probing ultrafast spin dynamics with optical pump-probe scanning tunnelling microscopy.," Nat. Nanotechnol., vol. 9, no. 8, pp. 588, 2014, https://doi.org/10.1038/nnano.2014.125.

[17] Z. H. Wang, C. H. Yoon, S. Yoshida, et al., "Surface-mediated spin dynamics probed by optical-pump-probe scanning tunneling microscopy.," Phys. Chem. Chem. Phys., vol. 21, no. 14, pp. 7256-7260, 2019, https://doi.org/10.1039/ с8ср07786j.

[18] A. M. Baro, R. Miranda, and J. L. Carrascosa, "Application to biology and technology of the scanning tunneling microscope operated in air at ambient pressure.," IBM J. Res. Dev., vol. 30, no. 4, pp. 380-386, 1986, https://doi.org/10.1147/rd.304. 0380 .

[19] D. R. T. Zahn, P. Tonndorf, R. Schmidt, et al., "Photoluminescence emission and Raman response of monolayer MoS2, MoSe2, and WSe2.," Optic Express, vol. 21, no. 4, pp. 4908-4916, 2013, https://doi.org/10.1364/oe.21. 004908.

[20] K. D. Park, O. Khatib, V. Kravtsov, G. Clark, X. Xu and M. B. Raschke, "Hybrid tip-enhanced nanospectroscopy and nanoimaging of monolayer WSe $\mathrm{S}_{2}$ with local strain control.," Nano Lett., vol. 16, no. 4, pp. 2621-2627, 2016, https://doi.org/10.1021/acs.nanolett. $6 \mathrm{~b} 00238$.

[21] A. Bouhelier, J. B. Renger, M. R. Beversluis, and L. Novotny, "Plasmon-coupled tip-enhanced near-field optical microscopy.," J. Microsc., vol. 210, no. 3, pp. 220-224, 2003, https://doi.org/10.1046/j.1365-2818.2003.01108.x.

[22] V. G. Dmitry and V. K. Vasily, "Optical properties of a plasmonic nano-antenna: an analytical approach.," New J. Phys., vol. 13, no. 5, p. 053034, 2011, https://doi.org/10.1046/j.1365-2818. 2003.01108.x.

[23] M. Born and E. Wolf, Principles of optics: Electromagnetic theory of propagation, interference and diffraction of light, 7th Edition, Amsterdam, Netherlands, Elsevier, 1999.

[24] E. H. Synge, "XXXVIII. A suggested method for extending microscopic resolution into the ultra-microscopic region.," Lond. Edinb. Dublin Phil. Mag. J. Sci., vol. 6, no. 35, pp. 356-362, 1928, https://doi.org/10.1080/14786440808564615.

[25] D. W. Pohl, W. Denk, and M. Lanz, “Optical stethoscopy: Image recording with resolution $\lambda / 20$.," Appl. Phys. Lett., vol. 44, no. 7, pp. 651-653, 1984, .

[26] A. Lewis, M. Isaacson, A. Harootunian, and A. Muray, “Development of a $500 \AA$ spatial resolution light microscope: I. light is efficiently transmitted through $\lambda / 16$ diameter apertures.," Ultramicroscopy, vol. 13, no. 3, pp. 227-231, 1984, https://doi.org/10.1016/0304-3991(84)90201-8.

[27] E. Betzig, J. K. Trautman, T. D. Harris, J. S. Weiner, and R. L. Kostelak, "Breaking the diffraction barrier: Optical microscopy on a nanometric scale.," Science, vol. 251, no. 5000, pp.1468-1470, 1991, https://doi. org/10.1126/science.251.5000.1468.

[28] U. Ch. Fischer and D. W. Pohl, "Observation of single-particle plasmons by near-field optical microscopy.," Phys. Rev. Lett., 
vol. 62 , no. 4, p. 458, 1989, https://doi.org/10.1103/ physrevlett.62.458.

[29] M. Specht, J. D. Pedarnig, W. M. Heckl, and T. W. Hänsch, "Scanning plasmon near-field microscope.," Phys. Rev. Lett., vol. 68 , no. 4, p. 476, 1992, https://doi.org/10.1103/ physrevlett.68.476.

[30] A. G. T. Ruiter, J. A. Veerman, M. F. Garcia-Parajo, and N. F. Van Hulst, "Single molecule rotational and translational diffusion observed by near-field scanning optical microscopy.," J. Phys. Chem. A, vol. 101, no. 40, pp. 7318-7323, 1997, https://doi.org/ 10.1021/jp971066s.

[31] D. A. Higgins, P. J. Reid, and P. F. Barbara, "Structure and exciton dynamics in J-aggregates studied by polarizationdependent near-field scanning optical microscopy.," J. Phys. Chem., vol. 100, no. 4, pp. 1174-1180, 1996, https://doi.org/ 10.1021/jp9518217.

[32] A. Lewis, A. Radko, N. B. Ami, D. Palanker, and K. Lieberman, "Near-field scanning optical microscopy in cell biology.," Trends Cell Biol., vol. 9, no. 2, pp. 70-73, 1999, https://doi.org/10. 1016/s0962-8924(98)01437-8.

[33] K. D. Park, M. B. Raschke, M. J. Jang, et al., "Near-field imaging of cell membranes in liquid enabled by active scanning probe mechanical resonance control.,"J. Phys. Chem. C, vol. 120, no. 37, pp. 21138-21144, 2016, https://doi.org/10.1021/acs.jpcc.6b06563.

[34] H. D. Hallen, A. H. La Rosa, and C. L. Jahncke, "Near-field scanning optical microscopy and spectroscopy for semiconductor characterization.," Phys. Status Solidi, vol. 152, no. 1, pp. 257-268, 1995, https://doi.org/10.1002/pssa. 2211520126.

[35] R. D. Grober, T. D. Harris, J. K. Trautman, et al., "Optical spectroscopy of a GaAs/AlGaAs quantum wire structure using near-field scanning optical microscopy.," Appl. Phys. Lett., vol. 64, no. 11, pp. 1421-1423, 1994, https://doi.org/10.1063/1.111903.

[36] M. S. Jeong, J. Y. Kim, Y. W. Kim, et al., "Spatially resolved photoluminescence in InGaN/GaN quantum wells by nearfield scanning optical microscopy.," Appl. Phys. Lett., vol. 79, no. 7, pp. 976-978, 2001, https://doi.org/10.1063/1. 1391227.

[37] J. M. Atkin, S. Berweger, A. C. Jones, and M. B. Raschke, "Nanooptical imaging and spectroscopy of order, phases, and domains in complex solids.," Adv. Phys., vol. 61, no. 6, pp. 745-842, 2012, https://doi.org/10.1080/00018732.2012.737982.

[38] H. A. Bethe, "Theory of diffraction by small holes.," Phys. Rev., vol. 66, no. 7-8, p. 163, 1944, https://doi.org/10.1103/physrev. 66.163.

[39] X. Shi, L. Hesselink, and R. L. Thornton, "Ultrahigh light transmission through a C-shaped nanoaperture.," Optic Lett., vol. 28 , no. 15, pp. 1320-1322, 2003, https://doi.org/10.1364/ ol.28.001320.

[40] Y. Inouye and S. Kawata, "Near-field scanning optical microscope with a metallic probe tip.," Optic Lett., vol. 19, no. 3, pp. 159-161, 1994, https://doi.org/10.1364/ol.19. 000159.

[41] F. Zenhausern, M. P. O' Boyle, and H. K. Wickramasinghe, "Apertureless near-field optical microscope.," Appl. Phys. Lett., vol. 65, no. 13, pp. 1623-1625, 1994, https://doi.org/10.1063/1. 112931.

[42] R. B. G. De Hollander, N. F. Van Hulst, and R. P. H. Kooyman, "Near field plasmon and force microscopy.," Ultramicroscopy, vol. 57, no. $2-3$, pp. 263-269, 1995, https://doi.org/10.1016/ 0304-3991(94)00150-l.

[43] Y. C. Martin, H. F. Hamann, and H. K. Wickramasinghe, "Strength of the electric field in aperture less near-field optical microscopy.," J. Appl. Phys., vol. 89, no. 10, pp. 5774-5778, 2001, https://doi.org/10.1063/1.1354655.

[44] R. Hillenbrand and F. Keilmann, "Material-specific mapping of metal/semiconductor/dielectric nanosystems at $10 \mathrm{~nm}$ resolution by backscattering near-field optical microscopy.," Appl. Phys. Lett., vol. 80, no. 1, pp. 25-27, 2002, https://doi.org/ 10.1063/1.1428767.

[45] K. Kneipp, Y. Wang, H. Kneipp, et al., "Single molecule detection using surface-enhanced Raman scattering (SERS).," Phys. Rev. Lett., vol. 78, no. 9, pp. 1667, 1997, https://doi.org/10.1103/ physrevlett.78.1667.

[46] S. Nie and S. R. Emory, "Probing single molecules and single nanoparticles by surface-enhanced Raman scattering.," Science, vol. 275, no. 5303, pp. 1102-1106, 1997, https://doi. org/10.1126/science.275.5303.1102.

[47] R. M. Stöckle, Y. D. Suh, V. Deckert, and R. Zenobi, "Nanoscale chemical analysis by tip-enhanced Raman spectroscopy.," Chem. Phys. Lett., vol. 318, no. 1-3, pp. 131-136, 2000, https:// doi.org/10.1016/s0009-2614(99)01451-7.

[48] M. S. Anderson, "Locally enhanced Raman spectroscopy with an atomic force microscope.," Appl. Phys. Lett., vol. 76, no. 21, pp. 3130-3132, 2000, https://doi.org/10.1063/1.126546.

[49] N. Hayazawa, Y. Inouye, Z. Sekkat, and S. Kawata, "Metallized tip amplification of near-field Raman scattering.," Optic Commun., vol. 183, no. 1-4, pp. 333-336, 2000, https://doi.org/10.1016/ s0030-4018(00)00894-4.

[50] Y. Fang, Z. Zhang, and M. Sun, “High vacuum tip-enhanced Raman spectroscope based on a scanning tunneling microscope.," Rev. Sci. Instrum., vol. 87, no. 3, p. 033104, 2016, https://doi.org/10.1063/1.4943291.

[51] Z. Zhang, S. Sheng, R. Wang, and M. Sun, "Tip-enhanced Raman spectroscopy.," Anal. Chem., vol. 88, no. 19, pp. 9328-9346, 2016, https://doi.org/10.1021/acs.analchem.6b02093.

[52] M. T. Wenzel, T. Härtling, P. Olk, et al., "Gold nanoparticle tips for optical field confinement in infrared scattering near-field optical microscopy.," Optic Express, vol. 16, no. 16, pp. 12302-12312, 2008, https://doi.org/10.1364/oe.16.012302.

[53] K. D. Park, M. B. Raschke, J. M. Atkin, Y. H. Lee, and M. S. Jeong, "Probing bilayer grain boundaries in large-area graphene with tip-enhanced Raman spectroscopy.," Adv. Mater., vol. 29, no. 7, 2017, Art no. 1603601, https://doi.org/10.1002/adma. 201603601.

[54] C. Zhang, B. Q. Chen, and Z. Y. Li, "Optical origin of subnanometer resolution in tip-enhanced Raman mapping.," J. Phys. Chem. C, vol. 119, no. 21, pp. 11858-11871, 2015, https:// doi.org/10.1021/acs.jpcc.5b02653.

[55] W. Su, N. Kumar, S. Mignuzzi, J. Crain, and D. Roy, "Nanoscale mapping of excitonic processes in single-layer $\mathrm{MoS}_{2}$ using tipenhanced photoluminescence microscopy.," Nanoscale, vol. 8, no. 20, pp. 10564-10569, 2016, https://doi.org/10.1039/ c5nr07378b.

[56] J. T. Krug, E. J. Sánchez, and X. S. Xie, "Fluorescence quenching in tip-enhanced nonlinear optical microscopy.," Appl. Phys. Lett., vol. 86 , no. 23, p. 233102, 2005, https://doi.org/10.1063/1. 1935769. 
[57] K. D. Park, and M. B. Raschke, "Polarization control with plasmonic antenna tips: A universal approach to optical nanocrystallography and vector-field imaging.," Nano Lett., vol. 18, no. 5, pp. 2912-2917, 2018, https://doi.org/10.1021/acs. nanolett.8b00108.

[58] H. Groß, J. M. Hamm, T. Tufarelli, O. Hess, and B. Hecht, "Nearfield strong coupling of single quantum dots.," Sci. Adv., vol. 4, no. 3, p. eaar4906, 2018, https://doi.org/10.1126/sciadv. aar4906.

[59] M. Böhmler, N. Hartmann, C. Georgi, et al., "Enhancing and redirecting carbon nanotube photoluminescence by an optical antenna.," Optic Express, vol. 18, no. 16, pp.16443-16451, 2010, https://doi.org/10.1364/oe.18.016443.

[60] K. D. Park, M. A. May, H. Leng, et al., "Tip-enhanced strong coupling spectroscopy, imaging, and control of a single quantum emitter.," Sci. Adv., vol. 5, no. 7, p. eaav5931, 2019, https://doi. org/10.1126/sciadv.aav5931.

[61] A. V. Ermushev, B. V. Mchedlishvili, V. A. Oleĭnikov, and A. V. Petukhov, "Surface enhancement of local optical fields and the lightning-rod effect.," Quant. Electron., vol. 23, no. 5, p. 435, 1993, https://doi.org/10.1070/qe1993v023n05abeh003090.

[62] V. M. Agranovich, Surface polaritons, Amsterdam, Netherlands, Elsevier, 2012.

[63] F. Keilmann and R. Hillenbrand, "Near-field microscopy by elastic light scattering from a tip.," Phil. Trans. Roy. Soc. Lond. Ser. A Math. Phys. Eng. Sci., vol. 362, no. 1817, pp. 787-805, 2004, https://doi.org/10.1098/rsta.2003.1347.

[64] M. B. Raschke and C. Lienau, "Apertureless near-field optical microscopy: Tip-sample coupling in elastic light scattering.," Appl. Phys. Lett., vol. 83, no. 24, pp. 5089-5091, 2003, https:// doi.org/10.1063/1.1632023.

[65] E. M Purcell, H. C. Torrey, and R. V. Pound, "Resonance absorption by nuclear magnetic moments in a solid.," Phys. Rev., vol. 69, no. 1-2, p. 37, 1946, https://doi.org/10.1103/physrev. 69.37.

[66] R. Carminati, J.-J. Greffet, C. Henkel, and J. M. Vigoureux, "Radiative and non-radiative decay of a single molecule close to a metallic nanoparticle.," Optic Commun., vol. 261, no. 2, pp. 368-375, 2006, https://doi.org/10.1016/j.optcom.2005.12.009.

[67] J. N. Farahani, D. W. Pohl, H. J. Eisler, and B. Hecht, "Single quantum dot coupled to a scanning optical antenna: A tunable superemitter.," Phys. Rev. Lett., vol. 95, no. 1, 2005, Art no. 017402, https://doi.org/10.1103/physrevlett.95.017402.

[68] C. D. Geddes, Surface plasmon enhanced, coupled and controlled fluorescence, New Jersey, United States, John Wiley \& Sons, 2017.

[69] K. D. Park, T. Jiang, G. Clark, X. Xu, and M. B. Raschke, "Radiative control of dark excitons at room temperature by nano-optical antenna-tip purcell effect.," Nat. Nanotechnol., vol. 13, no. 1, pp. 59-64, 2018, https://doi.org/10.1038/ s41565-017-0003-0.

[70] N. Behr and M. B. Raschke, "Optical antenna properties of scanning probe tips: plasmonic light scattering, tip-sample coupling, and near-field enhancement.," J. Phys. Chem. C, vol. 112, no. 10 , pp. 3766-3773, 2008, https://doi.org/10.1021/ jp7098009.

[71] D. S. Bulgarevich and M. Futamata, “Apertureless tip-enhanced Raman microscopy with confocal epi-illumination/collection optics.," Appl. Spectrosc., vol. 58, no. 7, pp. 757-761, 2004, https://doi.org/10.1366/0003702041389292.
[72] W. Zhang, X. Cui, and O. J. F. Martin, "Local field enhancement of an infinite conical metal tip illuminated by a focused beam.," J. Raman Spectrosc., vol. 40, no. 10, pp. 1338-1342, 2009, https://doi.org/10.1002/jrs.2439.

[73] A. V. Goncharenko, M. M. Dvoynenko, H. C. Chang, and J. K. Wang, "Electric field enhancement by a nanometer-scaled conical metal tip in the context of scattering-type near-field optical microscopy.," Appl. Phys. Lett., vol. 88, no. 10, p. 104101, 2006, https://doi.org/10.1063/1.2183362.

[74] J. Stadler, B. Oswald, T. Schmid, and R. Zenobi, “Characterizing unusual metal substrates for gap-mode tip-enhanced Raman spectroscopy.,"J. Raman Spectrosc., vol. 44, no. 2, pp. 227-233, 2013, https://doi.org/10.1002/jrs.4169.

[75] N. Kazemi-Zanjani, S. Vedraine, and F. Lagugné-Labarthet, "Localized enhancement of electric field in tip-enhanced Raman spectroscopy using radially and linearly polarized light.," Optic Express, vol. 21, no. 21, pp. 25271-25276, 2013, https://doi.org/ 10.1364/oe.21.025271.

[76] R. L. Olmon, B. Slovick, T. W. Johnson, et al., "Optical dielectric function of gold.," Phys. Rev. B, vol. 86, no. 23, p. 235147, 2012, https://doi.org/10.1103/physrevb.86.235147.

[77] F. Wang and Y. R. Shen, "General properties of local plasmons in metal nanostructures.," Phys. Rev. Lett., vol. 97, no. 20, p. 206806, 2006, https://doi.org/10.1103/physrevlett.97.206806.

[78] E. L. Ru and P. Etchegoin, Principles of surface-enhanced Raman Spectroscopy: And related plasmonic effects, Amsterdam, Netherlands, Elsevier, 2008.

[79] X. Huang and M. A. El-Sayed, "Gold nanoparticles: Optical properties and implementations in cancer diagnosis and photothermal therapy.," J. Adv. Res., vol. 1, no. 1, pp. 13-28, 2010, https://doi.org/10.1016/j.jare.2010.02.002.

[80] P. R. West, S. Ishii, G. V. Naik, N. K. Emani, V. M. Shalaev, and A. Boltasseva, "Searching for better plasmonic materials.," Laser Photon. Rev., vol. 4, no. 6, pp. 795-808, 2010, https://doi.org/ 10.1002/lpor.200900055.

[81] G. T. Boyd, Z. H. Yu, and Y. R. Shen, "Photoinduced luminescence from the noble metals and its enhancement on roughened surfaces.," Phys. Rev. B, vol. 33, no. 12, p. 7923, 1986, https:// doi.org/10.1103/physrevb.33.7923.

[82] L. Novotny and B. Hecht, Principles of nano-optics, Cambridge, United Kingdom, Cambridge University Press, 2012.

[83] R. M. Roth, N. C. Panoiu, M. M. Adams, R. M. Osgood, C. C. Neacsu, and M. B. Raschke, "Resonant-plasmon field enhancement from asymmetrically illuminated conical metallicprobe tips.," Optic Express, vol. 14, no. 7, pp. 2921-2931, 2006, https://doi.org/10.1364/oe.14.002921.

[84] A. Normatov, P. Ginzburg, N. Berkovitch, et al., "Efficient coupling and field enhancement for the nano-scale: Plasmonic needle.," Optic Express, vol. 18, no. 13, pp.14079-14086, 2010, https://doi.org/10.1364/oe.18.014079.

[85] L. Novotny, R. X. Bian, and X. S. Xie, "Theory of nanometric optical tweezers.,” Phys. Rev. Lett., vol. 79, no. 4, p. 645, 1997, https://doi.org/10.1103/physrevlett.79.645.

[86] R. G. Milner and D. Richards, "The role of tip plasmons in nearfield Raman microscopy.,”J. Microsc., vol. 202, no. 1, pp. 66-71, 2001, https://doi.org/10.1046/j.1365-2818.2001.00864.x.

[87] A. L. Demming, F. Festy, and D. Richards, "Plasmon resonances on metal tips: Understanding tip-enhanced Raman scattering.," J. Chem. Phys., vol. 122, no. 18, p. 184716, 2005, https://doi.org/ 10.1063/1.1896356. 
[88] M. Micic, N. Klymyshyn, Y. D. Suh, and H. P. Lu, "Finite element method simulation of the field distribution for AFM tipenhanced surface-enhanced Raman scanning microscopy.," J. Phys. Chem. B, vol. 107, no. 7, pp. 1574-1584, 2003, https:// doi.org/10.1021/jp022060s.

[89] C. A. Balanis, Antenna theory: analysis and design, New Jersey, United States, John Wiley \& Sons, 2016.

[90] M. Sun, Z. Zhang, L. Chen, S. Sheng, and H. Xu, "Plasmonic gradient effects on high vacuum tip-enhanced Raman spectroscopy.," Adv. Opt. Mat., vol. 2, no. 1, pp. 74-80, 2014, https://doi.org/10.1002/adom.201300296.

[91] M. Sun, Z. Zhang, L. Chen, et al., "Plasmon-driven selective reductions revealed by tip-enhanced Raman spectroscopy.," Adv. Mater. Interface, vol. 1, no. 5, p. 1300125, 2014, https:// doi.org/10.1002/admi.201300125.

[92] F. Lu, W. Zhang, L. Zhang, et al., "Nanofocusing of surface plasmon polaritons on metal-coated fiber tip under internal excitation of radial vector beam.," Plasmonics, vol. 14, no. 6, pp. 1593-1599, 2019, https://doi.org/10.1007/s11468-01900951-8.

[93] N. Hayazawa, A. Tarun, Y. Inouye, and S. Kawata, "Near-field enhanced Raman spectroscopy using side illumination optics.," J. Appl. Phys., vol. 92, no. 12, pp. 6983-6986, 2002, https://doi.org/10.1063/1.1519945.

[94] D. Mehtani, N. Lee, R. D. Hartschuh, et al., "Nano-Raman spectroscopy with side-illumination optics.," J. Raman Spectrosc., vol. 36, no. 11, pp. 1068-1075, 2005, https://doi. org/10.1002/jrs.1409.

[95] M. Sun, Y. Fang, Z. Zhang, and H. Xu, “Activated vibrational modes and Fermi resonance in tip-enhanced Raman spectroscopy.," Phys. Rev. E, vol. 87, no. 2, 2013, Art no. 020401, https://doi.org/10.1103/physreve.87.020401.

[96] M. Gao, M. Sun, and L. Meng, "Tip-enhanced spectroscopy of 2D black phosphorus.," J. Raman Spectrosc., vol. 50, no. 8, pp. 1058-1064, 2019, https://doi.org/10.1002/jrs.5610.

[97] D. Kurouski, S. Zaleski, F. Casadio, R. P. Van Duyne, and N. C. Shah, "Tip-enhanced Raman spectroscopy (TERS) for in situ identification of indigo and iron gall ink on paper.,"J. Am. Chem. Soc., vol. 136, no. 24, pp. 8677-8684, 2014, https://doi.org/ 10.1021/ja5027612.

[98] M. A. Lieb and A. J. Meixner, “A high numerical aperture parabolic mirror as imaging device for confocal microscopy.," Optic Express, vol. 8, no. 7, pp. 458-474, 2001, https://doi.org/ 10.1364/oe.8.000458.

[99] C. J. R. Sheppard and A. Choudhury, "Annular pupils, radial polarization, and superresolution.," Appl. Optic., vol. 43, no. 22, pp. 4322-4327, 2004, https://doi.org/10.1364/ao.43. 004322.

[100] J. Kim, D.C. Kim, and S.H. Back, "Demonstration of high lateral resolution in laser confocal microscopy using annular and radially polarized light.," Microsc. Res. Tech., vol. 72, no. 6, pp. 441-446, 2009, https://doi.org/10.1002/jemt.20689.

[101] W. T. Tang, E. Y. S. Yew, and C. J. R. Sheppard, "Polarization conversion in confocal microscopy with radially polarized illumination.," Optic Lett., vol. 34, no. 14, pp. 2147-2149, 2009, https://doi.org/10.1364/ol.34.002147.

[102] Y. Kozawa, T. Hibi, A. Sato, et al., "Lateral resolution enhancement of laser scanning microscopy by a higher-order radially polarized mode beam.," Optic Express, vol. 19, no. 17, pp. 15947-15954, 2011, https://doi.org/10.1364/oe.19.015947.
[103] J. Azoulay, A. Débarre, A. Richard, and P. Tchénio, "Field enhancement and apertureless near-field optical spectroscopy of single molecules.," J. Microsc., vol. 194, no. 2-3, pp. 486-490, 1999, https://doi.org/10.1046/j.1365-2818. 1999.00558.x.

[104] E. Betzig and R. J. Chichester, "Single molecules observed by near-field scanning optical microscopy.," Science, vol. 262, no. 5138, pp. 1422-1425, 1993, https://doi.org/10.1126/science. 262.5138.1422.

[105] T. Takagahara, "Enhanced excitonic optical nonlinearity and exciton dynamics in semiconductor microstructures.," Surf. Sci., vol. 196, no. 1-3, pp. 590-595, 1988, https://doi.org/10. 1016/0039-6028(88)90746-7.

[106] Z. Ma, J. M. Gerton, L. A. Wade, and S. R. Quake, "Fluorescence near-field microscopy of DNA at sub-10 nm resolution.," Phys. Rev. Lett., vol. 97, no. 26, 2006, https://doi.org/10.1103/ physrevlett.97.260801.

[107] H. G. Frey, J. Paskarbeit, and D. Anselmetti, “Tip-enhanced single molecule fluorescence near-field microscopy in aqueous environment.," Appl. Phys. Lett., vol. 94, no. 24, 2009, https:// doi.org/10.1063/1.3155190.

[108] C. Höppener and L. Novotny, “Antenna-based optical imaging of single $\mathrm{Ca}^{2+}$ transmembrane proteins in liquids.," Nano Lett., vol. 8, no. 2, pp. 642-646, 2008, https://doi.org/10.1021/ nl073057t.

[109] V. V. Protasenko, M. Kuno, A. Gallagher, and D. J. Nesbitt, "Fluorescence of single ZnS overcoated CdSe quantum dots studied by apertureless near-field scanning optical microscopy.," Optic Commun., vol. 210, no. 1-2, pp. 11-23, 2002, https://doi.org/10.1016/s00304018(02)01759-5.

[110] H. G. Frey, S. Witt, K. Felderer, and R. Guckenberger, “Highresolution imaging of single fluorescent molecules with the optical near-field of a metal tip.," Phys. Rev. Lett., vol. 93, no. 20, 2004, Art no. 200801, https://doi.org/10.1103/physrevlett. 93.200801.

[111] J. M. Gerton, L. A. Wade, G. A. Lessard, Z. Ma, and S. R. Quake, "Tip-enhanced fluorescence microscopy at 10 nanometer resolution.," Phys. Rev. Lett., vol. 93, no. 18, 2004, Art no. 180801, https://doi.org/10.1103/physrevlett.93.180801.

[112] W. E. Moerner and L. Kador, "Optical detection and spectroscopy of single molecules in a solid.," Phys. Rev. Lett., vol. 62, no. 21, pp. 2535-2538, 1989, https://doi.org/10.1103/ physrevlett.62.2535.

[113] X. S. Xie and R. C. Dunn, "Probing single molecule dynamics.," Science, vol. 265, no. 5170, pp. 361-364, 1994, https://doi.org/ 10.1126/science.265.5170.361.

[114] W. P. Ambrose, P. M. Goodwin, J. C. Martin, and R. A. Keller, "Single molecule detection and photochemistry on a surface using near-field optical excitation.," Phys. Rev. Lett., vol. 72, no. 1, pp. 160-163, 1994, https://doi.org/10.1103/physrevlett.72. 160.

[115] W. Denk and D. W Pohl, "Near-field optics: Microscopy with nanometer-size fields.," J. Vac. Sci. Technol. B, vol. 9, no. 2, pp. 510-513, 1991, https://doi.org/10.1116/1.585558.

[116] H. G. Frey, F. Keilmann, A. Kriele, and R. Guckenberger, "Enhancing the resolution of scanning near-field optical microscopy by a metal tip grown on an aperture probe.," Appl. Phys. Lett., vol. 81, no. 26, pp. 5030-5032, 2002, https://doi. org/10.1063/1.1530736. 
[117] C. Höppener and L. Novotny, "Imaging of membrane proteins using antenna-based optical microscopy.," Nanotechnology, vol. 19, no. 38, p. 384012, 2008, https://doi.org/10.1088/ 0957-4484/19/38/384012.

[118] J. Azoulay, A. Débarre, A. Richard, and P. Tchénio, "Quenching and enhancement of single-molecule fluorescence under metallic and dielectric tips.," Europhys. Lett., vol. 51, no. 4, p. 374, 2000, https://doi.org/10.1209/ epl/i2000-00504-y.

[119] V. V. Protasenko, A. Gallagher, and D. J. Nesbitt, “Factors that influence confocal apertureless near-field scanning optical microscopy.," Optic Commun., vol. 233, no. 1-3, pp. 45-56, 2004, https://doi.org/10.1016/j.optcom.2004.01.004.

[120] A. Hartschuh, E. J. Sánchez, X. S. Xie, and L. Novotny, “Highresolution near-field Raman microscopy of single-walled carbon nanotubes.," Phys. Rev. Lett., vol. 90, no. 9, 2003, Art no. 095503, https://doi.org/10.1103/physrevlett.90. 095503.

[121] E. J. Sánchez, L. Novotny, and X. S. Xie, "Near-field fluorescence microscopy based on two-photon excitation with metal tips.," Phys. Rev. Lett., 82, no. 20, pp. 4014-4017, 1999, https://doi. org/10.1103/physrevlett.82.4014.

[122] H. F. Hamann, M. Kuno, A. Gallagher, and D. J. Nesbitt, "Molecular fluorescence in the vicinity of a nanoscopic probe.," J. Chem. Phys., vol. 114, no. 19, pp. 8596-8609, 2001, https:// doi.org/10.1063/1.1365931.

[123] A. Kramer, W. Trabesinger, B. Hecht, and U. P. Wild, "Optical near-field enhancement at a metal tip probed by a single fluorophore.," Appl. Phys. Lett., vol. 80, no. 9, pp. 1652-1654, 2002, https://doi.org/10.1063/1.1453479.

[124] X. Dai, Z. Zhang, Y. Jin, et al., "Solution-processed, highperformance light-emitting diodes based on quantum dots.," Nature, vol. 515, no. 7525, pp. 96-99, 2014, https://doi.org/10. 1038 /nature13829.

[125] P. Michler, "A quantum dot single-photon turnstile device.," Science, vol. 290, no. 5500, pp. 2282-2285, 2000, https://doi. org/10.1126/science.290.5500.2282.

[126] J. J. Glennon, R. Tang, W. E. Buhro, and R. A. Loomis, "Synchronous photoluminescence intermittency (blinking) along whole semiconductor quantum wires.," Nano Lett., vol. 7, no. 11, pp. 3290-3295, 2007, https://doi.org/10.1021/ nl0714583.

[127] Y. Yan, C. Zhang, J. Yao, and Y. S. Zhao, "Recent advances in organic one-dimensional composite materials: Design, construction, and photonic elements for information processing.," Adv. Mater., vol. 25, no. 27, pp. 3627-3638, 2013, https://doi.org/10.1002/adma.201300325.

[128] H. Qian, P. T. Araujo, C. Georgi, et al., "Visualizing the local optical response of semiconducting carbon nanotubes to DNAwrapping.," Nano Lett., vol. 8, no. 9, pp. 2706-2711, 2008, https://doi.org/10.1021/nl801038t.

[129] H. Jin, E. S. Jeng, D. A. Heller, et al., "Divalent ion and thermally induced DNA conformational polymorphism on single-walled carbon nanotubes.," Macromolecules, vol. 40, no. 18 , pp. 6731-6739, 2007, https://doi.org/10.1021/ ma070608t.

[130] R. R. Johnson, A. T. C. Johnson, and M. L. Klein, "Probing the structure of DNA-carbon nanotube hybrids with molecular dynamics.," Nano Lett., vol. 8, no. 1, pp. 69-75, 2008, https:// doi.org/10.1021/nl071909j.
[131] S. Manohar, T. Tang, and A. Jagota, "Structure of homopolymer DNA-CNT hybrids.," J. Phys. Chem. C, vol. 111, no. 48, pp. 17835-17845, 2007, https://doi.org/10.1021/ jp071316x.

[132] M. Böhmler, Z. Wang, A. Myalitsin, A. Mews, and A. Hartschuh, "Optical imaging of CdSe nanowires with nanoscale resolution.," Angew. Chem. Int. Ed., vol. 50, no. 48, pp. 11536-11538, 2011, https://doi.org/10.1002/anie. 201105217.

[133] K. F. Mak, C. Lee, J. Hone, J. Shan, and T. F. Heinz, "Atomically thin $\mathrm{MoS}_{2}$ : A new direct-gap semiconductor.," Phys. Rev. Lett., vol. 105, no. 13, 2010, Art no. 136805, https://doi.org/10.1103/ physrevlett.105.136805.

[134] Q. H. Wang, K. Kalantar-Zadeh, A. Kis, J. N. Coleman, and M. S. Strano, "Electronics and optoelectronics of twodimensional transition metal dichalcogenides.," Nat. Nanotechnol., vol. 7, no. 11, p. 699, 2012, https://doi.org/ 10.1038/nnano.2012.193.

[135] A. Chernikov, T. C. Berkelbach, H. M. Hill, et al., "Exciton binding energy and nonhydrogenic Rydberg series in monolayer $\mathrm{WS}_{2}$.," Phys. Rev. Lett., vol. 113, no. 7, 2014, Art no. 076802, https:// doi.org/10.1103/physrevlett.113.076802.

[136] A. Boulesbaa, B. Huang, K. Wang, et al., "Observation of two distinct negative trions in tungsten disulfide monolayers.," Phys. Rev. B, vol. 92, no. 11, 2015, Art no. 115443, https://doi. org/10.1103/physrevb.92.115443.

[137] K. F. Mak and J. Shan, "Photonics and optoelectronics of 2D semiconductor transition metal dichalcogenides.," Nat. Photon., vol. 10, no. 4, p. 216, 2016, https://doi.org/10.1038/ nphoton.2015.282.

[138] P. K. Chow, R. B. Jacobs-Gedrim, J. Gao, et al., "Defect-induced photoluminescence in monolayer semiconducting transition metal dichalcogenides.," ACS Nano, vol. 9, no. 2, pp. 1520-1527, 2015, https://doi.org/10.1021/nn5073495.

[139] Z. Wu, W. Zhao, J. Jiang, et al., "Defect activated photoluminescence in WSe $\mathrm{W}_{2}$ monolayer.,"J. Phys. Chem. C, vol. 121, no. 22, pp. 12294-12299, 2017, https://doi.org/10.1021/ acs.jpcc.7b03585.

[140] L. Meng and M. Sun, "Tip-enhanced photoluminescence spectroscopy of monolayer MoSe 2 .," Photon. Res., vol. 5, no. 6, pp. 745-749, 2017, https://doi.org/10.1364/prj.5.000745.

[141] Y. Lee, S. Park, H. Kim, G. H. Han, Y. H. Lee, and J. Kim, "Characterization of the structural defects in CVD-grown monolayered $\mathrm{MoS}_{2}$ using near-field photoluminescence imaging.," Nanoscale, vol. 7, no. 28, pp. 11909-11914, 2015, https://doi.org/10.1039/c5nr02897c.

[142] W. Bao, N. J. Borys, C. Ko, et al., "Visualizing nanoscale excitonic relaxation properties of disordered edges and grain boundaries in monolayer molybdenum disulfide.," Nat. Commun., vol. 6, no. 1, pp. 1-7, 2015, https://doi.org/10.1038/ ncomms8993.

[143] A. M. Van Der Zande, P. Y. Huang, D. A. Chenet, et al., "Grains and grain boundaries in highly crystalline monolayer molybdenum disulphide.," Nat. Mater., vol. 12, no. 6, pp. 554-561, 2013, https://doi.org/10.1038/nmat3633.

[144] B. Liu, M. Fathi, L. Chen, A. Abbas, Y. Ma, and C. Zhou, "Chemical vapor deposition growth of monolayer WSe $\mathrm{W}_{2}$ with tunable device characteristics and growth mechanism study.," ACS Nano, vol. 9, no. 6, pp. 6119-6127, 2015, https://doi.org/ 10.1021/acsnano.5b01301. 
[145] C. Cong, J. Shang, X. Wu, et al., "Synthesis and optical properties of large-area single-crystalline $2 \mathrm{D}$ semiconductor $\mathrm{WS}_{2}$ monolayer from chemical vapor deposition.," Adv. Opt. Mater., vol. 2, no. 2, pp. 131-136, 2014, https://doi.org/10. 1002/adom.201300428.

[146] Y. Okuno, O. Lancry, A. Tempez, et al., "Probing the nanoscale light emission properties of a CVD-grown $\mathrm{MoS}_{2}$ monolayer by tip-enhanced photoluminescence.," Nanoscale, vol. 10, no. 29 , pp. 14055-14059, 2018, https://doi.org/10.1039/ c8nr02421a.

[147] S. Deng, A. V. Sumant, and V. Berry, "Strain engineering in twodimensional nanomaterials beyond graphene.," Nano Today, vol. 22, pp. 14-35, 2018, https://doi.org/10.1016/j.nantod.2018.07.001.

[148] S. T. Pantelides, "The electronic structure of impurities and other point defects in semiconductors.," Rev. Mod. Phys., vol. 50, no. 4, p. 797, 1978, https://doi.org/10.1103/revmodphys.50.797.

[149] Z. He, Z. Han, J. Yuan, et al., "Quantum plasmonic control of trions in a picocavity with monolayer WSe $e_{2}$," Sci. Adv., vol. 5, no. 10, p. eaau8763, 2019, https://doi.org/10.1126/sciadv.aau8763.
[150] G. Binnig and H. Rohrer, "Scanning tunneling microscopy.," Surf. Sci., 126, no. 1-3, pp. 236-244, 1983, https://doi.org/10. 1016/0039-6028(83)90716-1.

[151] J. Tersoff and D. R. Hamann, "Theory and application for the scanning tunneling microscope.," Phys. Rev. Lett., vol. 50, no. 25, p. 1998, 1983, https://doi.org/10.1103/physrevlett.50. 1998.

[152] G. Binnig, C. F. Quate, and Ch. Gerber, "Atomic force microscope.,” Phys. Rev. Lett., vol. 56, no. 9, p. 930, 1986, https://doi.org/10.1103/physrevlett.56.930.

[153] Y. Zhao, C. Riemersma, F. Pietra, R. Koole, C. de Mello Donegá, and A. Meijerink, "High-temperature luminescence quenching of colloidal quantum dots.," ACS Nano, vol. 6, no. 10, pp. 9058-9067, 2012, https://doi.org/10.1021/ nn303217q.

[154] M. Y. Shen, M. Oda, and T. Goto, "Direct evidence for photoionization in CdTe nanocrystals embedded in trioctylphosphine oxide.," Phys. Rev. Lett., vol. 82, no. 19, p. 3915, 1999, https://doi.org/10.1103/physrevlett.82.3915. 